\title{
HITTING TIME OF A HALF-LINE BY A TWO-DIMENSIONAL NON-SYMMETRIC RANDOM WALK
}

\author{
Yasunari FUKAI \\ (Received 9 April 2014 and revised 27 October 2014)
}

\begin{abstract}
We consider the probability that a two-dimensional random walk starting from the origin never returns to the half-line $(-\infty, 0] \times\{0\}$ before time $n$. Let $X=\left(X_{1}, X_{2}\right)$ be the increment of the two-dimensional random walk. For an aperiodic random walk with moment conditions $E\left[X_{2}\right]=0$ and $E\left[\left|X_{1}\right|^{\delta}\right]<\infty, E\left[\left|X_{2}\right|^{2+\delta}\right]<\infty$ for some $\delta \in(0,1)$, we obtain an asymptotic estimate (as $n \rightarrow \infty$ ) of this probability by assuming the behavior of the characteristic function of $X$ near zero.
\end{abstract}

\section{Introduction and statements of results}

We consider the probability $P_{n}$ that a two-dimensional random walk starting at the origin never returns to the half-line $(-\infty, 0] \times\{0\}$ before time $n$. The purpose of this paper is to investigate the asymptotic behavior of $P_{n}$ as $n \rightarrow \infty$.

For a simple random walk, $n^{1 / 4} P_{n}$ is bounded both from above and from below by positive constants. This bound was shown by Lawler [5, (2.35)]. The estimate plays an important role in computing an upper bound of a hitting distribution from infinity for a simple random walk (see [5, Proposition 2.4.10]). The upper bound gives a growth estimate for the two-dimensional diffusion limited aggregation model [4] and an estimate of the twodimensional intersection exponent [6].

Let $S=\{S(n)\}_{n=0}^{\infty}$ be the two-dimensional random walk starting at $x \in \mathbb{Z}^{2}$ defined by

$$
S(n)=x+X(1)+\cdots+X(n),
$$

where the $X(j)$ are independent copies of a $\mathbb{Z}^{2}$-valued random variable $X$. Here $S$ is called simple if $X$ takes the four values $(1,0),(-1,0),(0,1),(0,-1)$ with equal probability. We write $X=\left(X_{1}, X_{2}\right)$ and $S(n)=\left(S_{1}(n), S_{2}(n)\right)$. We denote by $P_{x}$ the distribution of the twodimensional random walk starting at $x$ and by $E_{x}$ its expectation. For a subset $A$ of $\mathbb{Z}^{2}$, define

$$
\tau_{A}=\inf \{n \geq 1: S(n) \in A\} .
$$

We also denote by $U$ the first coordinate axis:

$$
U=\left\{x=\left(x_{1}, x_{2}\right) \in \mathbb{Z}^{2}: x_{2}=0\right\}
$$

2010 Mathematics Subject Classification: Primary 60G50, 60E10.

Keywords: two-dimensional random walk; hitting probability; fluctuation identities; Fourier analysis.

(C) 2015 Faculty of Mathematics, Kyushu University 
and by $V_{-}$and $V_{+}$the left half and right half of $U$ defined by

$$
V_{-}=\left\{x=\left(x_{1}, x_{2}\right) \in \mathbb{Z}^{2}: x_{1} \leq 0, x_{2}=0\right\} .
$$

By replacing $\geq$ by $\leq$ in $V_{-}$, we define $V_{+}$. Here $S$ is called aperiodic if the smallest additive subgroup containing $\left\{x \in \mathbb{Z}^{2} \mid P\{X=x\}>0\right\}$ agrees with $\mathbb{Z}^{2}$.

THEOREM 1.1. Assume that $X$ satisfies the following:
(a) $S$ is aperiodic;
(b) $E\left[X_{2}\right]=0$;
(c) $E\left[\left|X_{1}\right|^{\delta}\right]<\infty$;
(d) $E\left[\left|X_{2}\right|^{2+\delta}\right]<\infty$ for some $\delta \in(0,1)$.

Assume that there exist constants $\alpha \in(0,2], \varepsilon>0$ and $c_{1}, c_{2} \geq 0$ with $c_{1}+c_{2}>0$ such that, as $\vartheta \rightarrow 0$,

$$
\begin{aligned}
& 1-E {\left[\cos \vartheta X_{1}\right]-\frac{\left(E\left[\left(\sin \vartheta X_{1}\right) X_{2}\right]\right)^{2}}{2 E\left[X_{2}^{2}\right]}+\frac{\left(E\left[\left(-1+\cos \vartheta X_{1}\right) X_{2}\right]\right)^{2}}{2 E\left[X_{2}^{2}\right]} } \\
&=c_{1}|\vartheta|^{\alpha}+O\left(|\vartheta|^{\alpha+\varepsilon}\right)
\end{aligned}
$$

and that, as $\vartheta \rightarrow 0$,

$$
\begin{gathered}
E\left[\sin \vartheta X_{1}\right]-\frac{E\left[\left(\sin \vartheta X_{1}\right) X_{2}\right] E\left[\left(-1+\cos \vartheta X_{1}\right) X_{2}\right]}{E\left[X_{2}^{2}\right]} \\
=c_{2}|\vartheta|^{\alpha} \frac{\vartheta}{|\vartheta|}+O\left(|\vartheta|^{\alpha+\varepsilon}\right) .
\end{gathered}
$$

Then

$$
P_{0}\left\{\tau_{V_{-}}>n\right\} \asymp n^{-1 / 4+\beta},
$$

where $\beta$ is the constant given by

$$
\beta=\frac{1}{\alpha \pi} \arcsin \frac{c_{2}}{\sqrt{2} \sqrt{c_{1}^{2}+c_{2}^{2}+c_{1} \sqrt{c_{1}^{2}+c_{2}^{2}}}},
$$

and we write $a_{n} \asymp b_{n}$ if $c, \tilde{c}>0$ such that $c b_{n} \leq a_{n} \leq \tilde{c} b_{n}$ for all $n$.

EXAMPLE 1.2. Suppose that (a), (b), (d), and (I), (II) below hold:

(I) $X_{1}$ and $X_{2}$ are independent;

(II) for $\alpha \in(1,2)$,

$$
P\left\{X_{1}=n\right\}= \begin{cases}c_{-} \frac{1}{|n|^{1+\alpha}} & n \in\{-1,-2, \ldots\}, \\ c_{+} & n=1, \\ 0 & \text { otherwise }\end{cases}
$$

with constants $c_{-}$and $c_{+}$satisfying

$$
\sum_{n \in \mathbb{Z}} P\left\{X_{1}=n\right\}=1 \quad \text { and } \quad \sum_{n \in \mathbb{Z}} n P\left\{X_{1}=n\right\}=0 .
$$


Indeed, by a simple calculation, we see that $X_{1}$ satisfies (c), and (1.1) and (1.2) become

$$
\begin{aligned}
1-E\left[\cos \vartheta X_{1}\right] & =\frac{c_{-} \pi}{2 \Gamma(\alpha+1) \cos ((\alpha-1) \pi / 2)}|\vartheta|^{\alpha}+O\left(|\vartheta|^{2}\right), \\
E\left[\sin \vartheta X_{1}\right] & =\frac{c_{-} \pi}{2 \Gamma(\alpha+1) \sin ((\alpha-1) \pi / 2)}|\vartheta|^{\alpha} \frac{\vartheta}{|\vartheta|}+O\left(|\vartheta|^{2}\right),
\end{aligned}
$$

where $\Gamma$ is the gamma function (see Appendix B). Then we deduce that $\beta=(2-\alpha) / 4 \alpha$ and

$$
P_{0}\left\{\tau_{V_{-}}>n\right\} \asymp n^{-(\alpha-1) /(2 \alpha)} .
$$

EXAMPLE 1.3. Suppose that $X$ satisfies the same condition as Example 1.2 except with (II) replaced by $P\left\{X_{1}=1\right\}=1$. Then it is clear that for $n \in \mathbb{N}$,

$$
P_{0}\left\{\tau_{V_{-}}>n\right\}=1
$$

(1.1) and (1.2) become

$$
1-E\left[\cos \vartheta X_{1}\right]=O\left(|\vartheta|^{2}\right), \quad E\left[\sin \vartheta X_{1}\right]=\vartheta+O\left(|\vartheta|^{2}\right) .
$$

Hence, we obtain

$$
P_{0}\left\{\tau_{V_{-}}>n\right\} \asymp 1,
$$

which is in agreement with $P_{0}\left\{\tau_{V_{-}}>n\right\}=1$.

Remark. (1) For a two-dimensional simple random walk, (1.3) was shown by Lawler [5, (2.35)] and Kesten [4]. Bousquet-Mélou and Schaeffer [1] considered $P_{0}\left\{\tau_{V_{-}}>n\right\}$ for a twodimensional random walk satisfying the condition that the increment $X$ takes finite values with equal probability. Moreover, in the case of a simple random walk, they gave a sharp estimate

$$
\lim _{n \rightarrow \infty} P_{0}\left\{\tau_{V_{-}}>n\right\} /\left(\frac{\sqrt{1+\sqrt{2}}}{2 \Gamma(3 / 4)} n^{-1 / 4}\right)=1
$$

by using a combinatorial argument. Isozaki [3] considered $P_{0}\left\{\tau_{V_{-}}>n\right\}$ for two-dimensional symmetric random walks.

(2) When $S$ is aperiodic with $E[X]=0$ and $E\left[|X|^{\delta}\right]<\infty$ for some $\delta>0$, it is proved that $n^{1 / 4} P_{0}\left\{\tau_{V_{-}}>n\right\}$ converges to some positive constant as $n \rightarrow \infty$ (see [2]). Although Theorem 1.1 provides a weaker result for this model than that in [2], it has the advantage that it can be applied to a large class of random walks.

Let $T_{\lambda}$ be a random variable independent of $S$ satisfying $P\left\{T_{\lambda}=j\right\}=(1-\lambda) \lambda^{j}(j \in$ $\{0\} \cup \mathbb{N}$ ). The key of the proof of Theorem 1.1 is to estimate $P_{0}\left\{T_{\lambda}<\tau_{V_{-}}\right\}$. In fact, by the Tauberian theorem, the asymptotic behavior of $P_{0}\left\{T_{\lambda}<\tau_{V_{-}}\right\}$as $\lambda \uparrow 1$ determines that of $P_{0}\left\{\tau_{V_{-}}>n\right\}$ as $n \rightarrow \infty$, which is our target in Theorem 1.1.

In [2], we deduce the asymptotic behavior of $P_{0}\left\{T_{\lambda}<\tau_{V_{-}}\right\}$as $\lambda \uparrow 1$ from that of $P_{0}\left\{T_{\lambda}<\right.$ $\left.\tau_{V_{-}}\right\} \times P_{0}\left\{T_{\lambda}<\tau_{V_{+}}\right\}$and $P_{0}\left\{T_{\lambda}<\tau_{V_{-}}\right\} / P_{0}\left\{T_{\lambda}<\tau_{V_{+}}\right\}$. The method to control $P_{0}\left\{T_{\lambda}<\tau_{V_{-}}\right\}$ follows from [2]. We need a new device to estimate $P_{0}\left\{T_{\lambda}<\tau_{V_{-}}\right\} / P_{0}\left\{T_{\lambda}<\tau_{V_{+}}\right\}$.

The organization of the paper is as follows. In Section 2, we quote some results from [2]. In Section 3, we state some results needed to prove Theorem 1.1. In Section 4, we give a proof of Theorem 1.1. In Appendix A, we give the proof of lemmas not proved in Section 3. 


\section{Fundamental relations}

LEMMA 2.1. For an arbitrary two-dimensional random walk,

$$
\begin{aligned}
P_{0}\left\{T_{\lambda}<\tau_{U}\right\} & =P_{0}\left\{T_{\lambda}<\tau_{\tilde{V}_{+}}\right\} P_{0}\left\{T_{\lambda}<\tau_{V_{-}}\right\} \\
& =\left(1-P_{0}\left\{S\left(\tau_{V_{+}}\right)=0, \tau_{V_{+}} \leq T_{\lambda}\right\}\right)^{-1} P_{0}\left\{T_{\lambda}<\tau_{V_{+}}\right\} P_{0}\left\{T_{\lambda}<\tau_{V_{-}}\right\},
\end{aligned}
$$

where $\tilde{V}_{+}=V_{+} \backslash\{0\}$.

The proof of Lemma 2.1 is identical to that given for Proposition 2.4.6 in [5] and is thus omitted (see [2, Lemma 2.1]).

LeMmA 2.2. A two-dimensional random walk satisfying (a), (b) and $E\left[X_{2}^{2}\right]<\infty$ has the property that

$$
P_{0}\left\{T_{\lambda}<\tau_{U}\right\} \sim\left(2 E\left[X_{2}^{2}\right]\right)^{1 / 2}(1-\lambda)^{1 / 2},
$$

where we write $\alpha_{\lambda} \sim \beta_{\lambda}$ if $\lim _{\lambda \uparrow 1} \alpha_{\lambda} / \beta_{\lambda}=1$.

The proof of Lemma 2.2 is identical to that given in $[\mathbf{5}$, p. 68] and is thus omitted (see [2, Proposition 3.1]).

To formulate the next lemma, we introduce some notation. For $S$, we introduce the following random variables:

$$
\begin{aligned}
& \eta(1)=\inf \{j \geq 1 \mid S(j) \in U\}, \quad \eta(k+1)=\inf \{j>\eta(k) \mid S(j) \in U\} \quad(k=1,2, \ldots), \\
& \zeta(1)=S_{1}(\eta(1)), \quad \zeta(k+1)=S_{1}(\eta(k+1)) \quad(k=1,2, \ldots) .
\end{aligned}
$$

For $\lambda \in(0,1)$, we define

$$
\begin{gathered}
c(\lambda)=\exp \left(-\sum_{k=1}^{\infty} \frac{1}{k} E_{0}\left[\lambda^{\eta(k)} ; \zeta(k)=0\right]\right), \\
f_{\infty}(z ; \lambda)=\exp \left(-\sum_{k=1}^{\infty} \frac{1}{k} E_{0}\left[\lambda^{\eta(k)} z^{\zeta(k)} ; \zeta(k)<0\right]\right), \\
f_{0}(z ; \lambda)=\exp \left(-\sum_{k=1}^{\infty} \frac{1}{k} E_{0}\left[\lambda^{\eta(k)} z^{\zeta(k)} ; \zeta(k)>0\right]\right) .
\end{gathered}
$$

LeMma 2.3. For every two-dimensional random walk satisfying (b) and $E\left[X_{2}^{2}\right]<\infty$,

$$
\begin{aligned}
& P_{0}\left\{T_{\lambda}<\tau_{V_{-}}\right\}=c(\lambda) f_{\infty}(1 ; \lambda), \\
& P_{0}\left\{T_{\lambda}<\tau_{V_{+}}\right\}=c(\lambda) f_{0}(1 ; \lambda) .
\end{aligned}
$$

Moreover, for $l \in \mathbb{Z}$,

$$
E_{0}\left[\lambda^{\eta(k)} ; \zeta(k)=l\right]=\frac{1}{2 \pi} \int_{-\pi}^{\pi} e^{-i \theta_{1} l}\left(1-\left(\frac{1}{2 \pi} \int_{-\pi}^{\pi} \frac{1}{1-\lambda \phi(\theta)} d \theta_{2}\right)^{-1}\right)^{k} d \theta_{1},
$$

where $\phi(\theta)$ is the characteristic function of $X$, that is,

$$
\phi(\theta)=E[\exp (i \theta \cdot X)], \quad \theta=\left(\theta_{1}, \theta_{2}\right) \in \mathbb{R}^{2} .
$$

The proof of Lemma 2.3 is identical to that given in Section 17, Proposition 5 of [7] and is thus omitted (see [2, Proposition 2.4]). 


\section{Preliminary lemmas}

Let $a_{\lambda}\left(\theta_{1}\right)$ and $b_{\lambda}\left(\theta_{1}\right)$ be real numbers such that

$$
\frac{1}{2 \pi} \int_{-\pi}^{\pi} \frac{1}{1-\lambda \phi(\theta)} d \theta_{2}=a_{\lambda}\left(\theta_{1}\right)+i b_{\lambda}\left(\theta_{1}\right) .
$$

Here we write $\theta=\left(\theta_{1}, \theta_{2}\right)$. Let

$$
\begin{aligned}
\varphi_{\lambda}(\theta)= & 1-\lambda E\left[\cos \theta_{1} X_{1}\right]+\lambda E\left[\left(\sin \theta_{1} X_{1}\right) X_{2}\right] \theta_{2}+\frac{\lambda}{2} E\left[X_{2}^{2}\right] \theta_{2}^{2} \\
& -i\left\{\lambda E\left[\sin \theta_{1} X_{1}\right]+\lambda E\left[\left(-1+\cos \theta_{1} X_{1}\right) X_{2}\right] \theta_{2}\right\} .
\end{aligned}
$$

Then we have the following lemma.

LEMMA 3.1. Assume that $(a)-(d)$ hold. Then there exists a constant $c_{3}>0$ such that, for $\lambda \in(1 / 2,1)$ and $\theta \in[-\pi, \pi]^{2}$,

$$
\left|\frac{\varphi_{\lambda}(\theta)}{1-\lambda \phi(\theta)}-1\right| \leq c_{3}\left(\left|\theta_{1}\right|^{\hat{\delta}}+\left|\theta_{2}\right|^{\delta}\right),
$$

where $\hat{\delta}=\delta^{2} /(2+\delta)$. In addition, there exist $\tilde{c}_{3}>0$ and $r_{0}>0$ such that,

$$
\left|\frac{\Re[1-\lambda \phi(\theta)]}{\Re\left[\varphi_{\lambda}(\theta)\right]}-1\right| \leq \tilde{c}_{3}\left(\left|\theta_{1}\right|^{\hat{\delta}}+\left|\theta_{2}\right|^{\delta}\right)
$$

for $\lambda \in(1 / 2,1)$ and $\theta \in[-\pi, \pi]^{2}$ with $|\theta|<r_{0}$.

Proof. By definition, $1-\lambda \phi(\theta)=1-\lambda E\left[e^{i \theta_{1} X_{1}} e^{i \theta_{2} X_{2}}\right]$. We use Taylor polynomials for $\cos x$ and $\sin x$ at $x=0$ to obtain

$$
\left|e^{i x}-\left(1+i x-\frac{1}{2} x^{2}\right)\right| \leq 2 \min \left\{x^{2},|x|^{3}\right\} \leq 2|x|^{2+\delta} \quad(x \in \mathbb{R}) .
$$

This inequality and condition $(\mathrm{d})$ give

$$
\left|E\left[e^{i \theta_{1} X_{1}} e^{i \theta_{2} X_{2}}\right]-E\left[e^{i \theta_{1} X_{1}}\left(1+i \theta_{2} X_{2}-\frac{1}{2} \theta_{2}^{2} X_{2}^{2}\right)\right]\right| \leq 2 E\left[\left|X_{2}\right|^{2+\delta}\right]\left|\theta_{2}\right|^{2+\delta} .
$$

Using the Hölder inequality and conditions (c) and (d), we obtain

$$
E\left[\left|X_{1}\right|^{\hat{\delta}} X_{2}^{2}\right]<\infty .
$$

The inequality $\left|e^{i x}-1\right| \leq 3 \min \{1,|x|\} \leq 3|x|^{\hat{\delta}}(x \in \mathbb{R})$ gives

$$
\left|E\left[e^{i \theta_{1} X_{1}} X_{2}^{2}\right]-E\left[X_{2}^{2}\right]\right| \leq 3 E\left[\left|X_{1}\right|^{\hat{\delta}} X_{2}^{2}\right]\left|\theta_{1}\right|^{\hat{\delta}} .
$$

We note that

$$
E\left[\left(-1+\cos \theta_{1} X_{1}\right) X_{2}\right]=E\left[\left(\cos \theta_{1} X_{1}\right) X_{2}\right]
$$

from condition (b). From (3.3), (3.4), (3.5), we deduce that

$$
\left|1-\lambda \phi(\theta)-\varphi_{\lambda}(\theta)\right| \leq c\left(\left|\theta_{1}\right|^{\hat{\delta}}+\left|\theta_{2}\right|^{\delta}\right) \theta_{2}^{2}
$$

for some suitable constant $c>0$. Since the random walk is aperiodic, there exists a constant $c_{*}>0$ such that, for $\lambda \in(1 / 2,1)$ and $\theta \in[-\pi, \pi]^{2}$,

$$
|1-\lambda \phi(\theta)| \geq \Re[1-\lambda \phi(\theta)] \geq \lambda(1-\Re[\phi(\theta)]) \geq \frac{1}{2} c_{*}|\theta|^{2}
$$

as shown in [7, Section 7, Proposition 5]. Equation (3.1) follows from (3.6) and (3.7). 
Equation (3.6) implies that

$$
\left|\Re[1-\lambda \phi(\theta)]-\Re\left[\varphi_{\lambda}(\theta)\right]\right| \leq c\left(\left|\theta_{1}\right|^{\hat{\delta}}+\left|\theta_{2}\right|^{\delta}\right) \theta_{2}^{2} .
$$

By combining (3.8) with the last inequality in (3.7), there exists $r_{0}>0$ (small enough) such that, for $\lambda \in(1 / 2,1)$ and $\theta \in[-\pi, \pi]^{2}$ with $|\theta|<r_{0}$,

$$
\Re\left[\varphi_{\lambda}(\theta)\right] \geq \frac{1}{4} c_{*} \theta_{2}^{2} .
$$

Equation (3.2) follows from (3.8) and (3.9).

To estimate $a_{\lambda}(\theta)$, we calculate

$$
\frac{1}{2 \pi} \int_{-r_{0} / 2}^{r_{0} / 2} \Re\left[\frac{1}{\varphi_{\lambda}(\theta)}\right] d \theta_{2},
$$

where $r_{0}$ is the same constant as in Lemma 3.1. Let

$$
\begin{aligned}
& A=\frac{E\left[\left(\sin \theta_{1} X_{1}\right) X_{2}\right]}{(1 / 2) E\left[X_{2}^{2}\right]}, \quad B=\frac{1-\lambda E\left[\cos \theta_{1} X_{1}\right]}{(\lambda / 2) E\left[X_{2}^{2}\right]}, \\
& C=\frac{E\left[\left(-1+\cos \theta_{1} X_{1}\right) X_{2}\right]}{(1 / 2) E\left[X_{2}^{2}\right]}, \quad D=\frac{E\left[\sin \theta_{1} X_{1}\right]}{(1 / 2) E\left[X_{2}^{2}\right]} .
\end{aligned}
$$

Then

$$
\varphi_{\lambda}(\theta)=\frac{\lambda}{2} E\left[X_{2}^{2}\right]\left\{\theta_{2}^{2}+A \theta_{2}+B-i\left(C \theta_{2}+D\right)\right\}
$$

and, for each $\theta_{1}$,

$$
\Re\left[\frac{1}{\varphi_{\lambda}(\theta)}\right]=\frac{\Re\left[\varphi_{\lambda}(\theta)\right]}{\left|\varphi_{\lambda}(\theta)\right|^{2}}
$$

is a rational function in $\theta_{2}$.

Put

$$
K=\left(4 B-A^{2}+C^{2}\right)^{2}+4(2 D-A C)^{2} .
$$

To calculate the integral of $\Re\left[1 / \varphi_{\lambda}(\theta)\right]$ over $\left[-r_{0} / 2, r_{0} / 2\right]$ with respect to $\theta_{2}$, we use routine integration of a rational function using partial fractions. This calculation gives the following lemma.

Lemma 3.2. Suppose that $0<E\left[X_{2}^{2}\right]<\infty$. Then there exist $c_{4}>0, \lambda_{*} \in(1 / 2,1)$ and $s_{*}>$ 0 such that, for all $\lambda \in\left(\lambda_{*}, 1\right)$ and $\theta_{1} \in[-\pi, \pi]$ with $0<\left|\theta_{1}\right|<s_{*}$,

$$
\left|\frac{1}{2 \pi} \int_{-r_{0} / 2}^{r_{0} / 2} \Re\left[\frac{1}{\varphi_{\lambda}(\theta)}\right] d \theta_{2}-\tilde{a}_{\lambda}\left(\theta_{1}\right)\right| \leq c_{4},
$$

where

$$
\tilde{a}_{\lambda}\left(\theta_{1}\right)=\frac{\sqrt{2}}{\lambda E\left[X_{2}^{2}\right] \sqrt{K}} \sqrt{4 B-A^{2}+C^{2}+\sqrt{K}} .
$$

From (3.1), (3.6), (3.7) and (3.9), we deduce the following. 
Lemma 3.3. Suppose that $(a)-(d)$. Then there exist $c_{5}>0, \lambda_{*} \in(1 / 2,1)$ and $s_{*}>0$ such that, for $\lambda \in\left(\lambda_{*}, 1\right)$ and $\theta_{1} \in[-\pi, \pi]$ with $0<\left|\theta_{1}\right|<s_{*}$,

$$
\left|a_{\lambda}\left(\theta_{1}\right)-\frac{1}{2 \pi} \int_{-r_{0} / 2}^{r_{0} / 2} \Re\left[\frac{1}{\varphi_{\lambda}(\theta)}\right] d \theta_{2}\right| \leq c_{5}\left(\left|\theta_{1}\right|^{\hat{\delta}}+K^{\delta / 4}|\log K|\right) K^{-1 / 4} .
$$

LEMmA 3.4. Suppose that $0<E\left[X_{2}^{2}\right]<\infty$. Then there exist $c_{6}>0, \lambda_{*} \in(1 / 2,1)$ and $s_{*}>$ 0 such that, for all $\lambda \in\left(\lambda_{*}, 1\right)$ and $\theta_{1} \in(-\pi, \pi)$ with $0<\left|\theta_{1}\right|<s_{*}$,

$$
\left|\frac{1}{2 \pi} \int_{-r_{0} / 2}^{r_{0} / 2} \Im\left[\frac{1}{\varphi_{\lambda}(\theta)}\right] d \theta_{2}-\tilde{b}_{\lambda}\left(\theta_{1}\right)\right| \leq c_{6}\left(\frac{|2 D-A C|}{\sqrt{K}}+\frac{|C|}{K^{1 / 4}}\right),
$$

where

$$
\tilde{b}_{\lambda}\left(\theta_{1}\right)=\frac{\sqrt{2}}{\lambda E\left[X_{2}^{2}\right] \sqrt{K}} \frac{2(2 D-A C)}{\sqrt{4 B-A^{2}+C^{2}+\sqrt{K}}} .
$$

Lemma 3.5. Suppose that $(a)-(d)$ hold. Then there exist $c_{7}>0, \lambda_{*} \in(1 / 2,1)$ and $s_{*}>0$ such that, for $\lambda \in\left(\lambda_{*}, 1\right)$ and $\theta_{1} \in[-\pi, \pi]$ with $0<\left|\theta_{1}\right|<s_{*}$,

$$
\begin{aligned}
& \left|b_{\lambda}\left(\theta_{1}\right)-\frac{1}{2 \pi} \int_{-r_{0} / 2}^{r_{0} / 2} \Im\left[\frac{1}{\varphi_{\lambda}(\theta)}\right] d \theta_{2}\right| \\
& \quad \leq c_{7}\left\{\left|\theta_{1}\right|^{\hat{\delta}}+|A| K^{(-1+\delta) / 4}+\left(\left|\theta_{1}\right|^{\hat{\delta}}+K^{\delta / 4}\right)\left(|C|+|D| K^{-1 / 4}\right) K^{-1 / 4}\right\} K^{-1 / 4} .
\end{aligned}
$$

The proofs of the above four lemmas are given in Appendix A.

\section{Proof of Theorem 1.1}

To prove Theorem 1.1, we consider $P_{0}\left\{T_{\lambda}<\tau_{V_{-}}\right\} / P_{0}\left\{T_{\lambda}<\tau_{V_{+}}\right\}$.

LEMMA 4.1. Suppose that (b) holds and $E\left[X_{2}^{2}\right]<\infty$. Then

$$
\frac{P_{0}\left\{T_{\lambda}<\tau_{V_{-}}\right\}}{P_{0}\left\{T_{\lambda}<\tau_{V_{+}}\right\}}=\frac{f_{\infty}(1 ; \lambda)}{f_{0}(1 ; \lambda)}=\exp \left(\lim _{L \rightarrow \infty} C_{L}(\lambda)\right)
$$

where

$$
C_{L}(\lambda)=\frac{1}{2 \pi} \int_{-\pi}^{\pi}\left\{\frac{\sin \vartheta}{1-\cos \vartheta}(1-\cos L \vartheta)+\sin L \vartheta\right\} \arcsin \frac{b_{\lambda}(\vartheta)}{\sqrt{a_{\lambda}^{2}(\vartheta)+b_{\lambda}^{2}(\vartheta)}} d \vartheta
$$

Proof. By Lemma 2.3 and the definitions of $f_{0}(z ; \lambda)$ and $f_{\infty}(z ; \lambda)$, we have

$$
\frac{P_{0}\left\{T_{\lambda}<\tau_{V_{-}}\right\}}{P_{0}\left\{T_{\lambda}<\tau_{V_{+}}\right\}}=\frac{f_{\infty}(1 ; \lambda)}{f_{0}(1 ; \lambda)}=\exp \left(\lim _{L \rightarrow \infty} C_{L}(\lambda)\right)
$$

where

$$
C_{L}(\lambda)=\sum_{k=1}^{\infty} \frac{1}{k} \sum_{l=1}^{L}\left(E_{0}\left[\lambda^{\eta(k)} ; \zeta(k)=l\right]-E_{0}\left[\lambda^{\eta(k)} ; \zeta(k)=-l\right]\right) .
$$

To obtain an expression for $C_{L}(\lambda)$, we use (2.1) in Lemma 2.3 which implies that

$$
C_{L}(\lambda)=\Re\left[\sum_{k=1}^{\infty} \frac{1}{2 \pi} \int_{-\pi}^{\pi} e_{L}\left(\theta_{1}\right) \frac{1}{k}\left(1-\left(\frac{1}{2 \pi} \int_{-\pi}^{\pi} \frac{1}{1-\lambda \phi(\theta)} d \theta_{2}\right)^{-1}\right)^{k} d \theta_{1}\right],
$$


where

$$
e_{L}\left(\theta_{1}\right)=\sum_{l=1}^{L}\left(e^{-i l \theta_{1}}-e^{i l \theta_{1}}\right) .
$$

From the inequality [2, pp. 332-333]

$$
\sup _{\theta_{1} \in[-\pi, \pi]}\left|1-\left(\frac{1}{2 \pi} \int_{-\pi}^{\pi} \frac{1}{1-\lambda \phi(\theta)} d \theta_{2}\right)^{-1}\right|<1,
$$

we interchange the order of summation and integration on the right-hand side of (4.3), and then use the identity

$$
-\sum_{k=1}^{\infty} \frac{z^{k}}{k}=\log (1-z) \quad(|z|<1) .
$$

Here, $\log (z)$ is the principal $\operatorname{logarithm}$ of $z$ and we choose $|\Im[\log (z)]|<\pi$. Here $C_{L}(\lambda)$ reduces to

$$
\frac{1}{2 \pi} \int_{-\pi}^{\pi} e_{L}\left(\theta_{1}\right) \log \left(\frac{1}{2 \pi} \int_{-\pi}^{\pi} \frac{1}{1-\lambda \phi(\theta)} d \theta_{2}\right) d \theta_{1} .
$$

By a simple calculation,

$$
e_{L}\left(\theta_{1}\right)=i\left\{\frac{\sin \theta_{1}}{1-\cos \theta_{1}}\left(-1+\cos L \theta_{1}\right)-\sin L \theta_{1}\right\} .
$$

If $\Re[z]>0$, then $\Im[\log (z)] \in(-\pi / 2, \pi / 2)$ and

$$
\Im[\log (z)]=\arcsin \frac{\Im[z]}{|z|} .
$$

Recall that $a_{\lambda}\left(\theta_{1}\right)$ is the real part of $(1 /(2 \pi)) \int_{-\pi}^{\pi} 1 /(1-\lambda \phi(\theta)) d \theta_{2}$ and $b_{\lambda}\left(\theta_{1}\right)$ the imaginary part. It is easy to check that $a_{\lambda}\left(\theta_{1}\right)>0$. Thus, we have (4.2).

Let

$$
\begin{aligned}
& B(\vartheta)=1-E\left[\cos \vartheta X_{1}\right]-\frac{\left(E\left[\left(\sin \vartheta X_{1}\right) X_{2}\right]\right)^{2}}{2 E\left[X_{2}^{2}\right]}+\frac{\left(E\left[\left(-1+\cos \vartheta X_{1}\right) X_{2}\right]\right)^{2}}{2 E\left[X_{2}^{2}\right]}, \\
& D(\vartheta)=E\left[\sin \vartheta X_{1}\right]-\frac{E\left[\left(\sin \vartheta X_{1}\right) X_{2}\right] E\left[\left(-1+\cos \vartheta X_{1}\right) X_{2}\right]}{E\left[X_{2}^{2}\right]}
\end{aligned}
$$

and

$$
K_{\lambda}(\vartheta)=(1-\lambda+\lambda B(\vartheta))^{2}+(\lambda D(\vartheta))^{2} .
$$

Then $\tilde{a}_{\lambda}$ in Lemma 3.2 can be written as

$$
\tilde{a}_{\lambda}(\vartheta)=\frac{1}{2 \sqrt{\lambda E\left[X_{2}^{2}\right]} K_{\lambda}(\vartheta)^{1 / 2}} \sqrt{1-\lambda+\lambda B(\vartheta)+K_{\lambda}(\vartheta)^{1 / 2}},
$$

and $\tilde{b}_{\lambda}$ in Lemma 3.4 can be written as

$$
\tilde{b}_{\lambda}(\vartheta)=\frac{1}{2 \sqrt{\lambda E\left[X_{2}^{2}\right]} K_{\lambda}(\vartheta)^{1 / 2}} \frac{\lambda D(\vartheta)}{\sqrt{1-\lambda+\lambda B(\vartheta)+K_{\lambda}(\vartheta)^{1 / 2}}} .
$$

Hence,

$$
\frac{\tilde{b}_{\lambda}(\vartheta)}{\sqrt{\tilde{a}_{\lambda}^{2}(\vartheta)+\tilde{b}_{\lambda}^{2}(\vartheta)}}=\frac{\lambda D(\vartheta)}{\sqrt{2} \sqrt{K_{\lambda}(\vartheta)+(1-\lambda+\lambda B(\vartheta)) K_{\lambda}(\vartheta)^{1 / 2}}} .
$$

To prove (1.3) in Theorem 1.1 for $c_{1}>0$ and $c_{2}>0$, we assume the following condition. 
Assumption (A1). There exist constants $\alpha \in(0,2]$ and $\varepsilon, c_{1}, c_{2}>0$ such that

$$
B(\vartheta)=c_{1}|\vartheta|^{\alpha}+O\left(|\vartheta|^{\alpha+\varepsilon}\right) \quad(\vartheta \rightarrow 0)
$$

and

$$
D(\vartheta)=c_{2}|\vartheta|^{\alpha} \frac{\vartheta}{|\vartheta|}+O\left(|\vartheta|^{\alpha+\varepsilon}\right) \quad(\vartheta \rightarrow 0)
$$

From assumption (A1) and the inequality

$$
\left|\sqrt{x+y}-\sqrt{x^{\prime}+y^{\prime}}\right| \leq\left|\sqrt{x}-\sqrt{x^{\prime}}\right|+\left|\sqrt{y}-\sqrt{y^{\prime}}\right| \quad\left(x, y, x^{\prime}, y^{\prime}>0\right),
$$

it is easy to see that there exist constants $c_{8}>0, s>0$ such that, for $\lambda \in(1 / 2,1)$ and $0<|\vartheta| \leq s$

$$
\begin{aligned}
\left|K_{\lambda}(\vartheta)^{1 / 2}-k_{\lambda}(\vartheta)^{1 / 2}\right| & =\left|\sqrt{(1-\lambda+\lambda B(\vartheta))^{2}+(\lambda D(\vartheta))^{2}}-k_{\lambda}(\vartheta)^{1 / 2}\right| \\
& \leq\left.\left|B(\vartheta)-c_{1}\right| \vartheta\right|^{\alpha}|+| D(\vartheta)-\left.c_{2}|\vartheta|^{\alpha} \frac{\vartheta}{|\vartheta|}\left|\leq c_{8} k_{\lambda}(\vartheta)^{1 / 2}\right| \vartheta\right|^{\varepsilon}
\end{aligned}
$$

where

$$
k_{\lambda}(\vartheta)=\left(1-\lambda+\lambda c_{1}|\vartheta|^{\alpha}\right)^{2}+\left(\lambda c_{2}|\vartheta|^{\alpha} \frac{\vartheta}{|\vartheta|}\right)^{2} .
$$

Equation (4.5) implies the following lemma.

Lemma 4.2. Assume that $0<E\left[X_{2}^{2}\right]<\infty$ and (A1) hold. Then there exist $c_{9}>0, s_{*}>0$ such that, for $\lambda \in(1 / 2,1)$ and $0<|\vartheta| \leq s_{*}$,

$$
\left|\frac{\tilde{b}_{\lambda}(\vartheta)}{\sqrt{\tilde{a}_{\lambda}^{2}(\vartheta)+\tilde{b}_{\lambda}^{2}(\vartheta)}}-\frac{\lambda c_{2}|\vartheta|^{\alpha}(\vartheta /|\vartheta|)}{\sqrt{2} Q_{\lambda}\left(|\vartheta|^{\alpha}\right)}\right| \leq c_{9}|\vartheta|^{\varepsilon},
$$

where

$$
Q_{\lambda}(s)=\left\{\left(1-\lambda+\lambda c_{1} s\right)^{2}+\left(\lambda c_{2} s\right)^{2}+\left(1-\lambda+\lambda c_{1} s\right) \sqrt{\left(1-\lambda+\lambda c_{1} s\right)^{2}+\left(\lambda c_{2} s\right)^{2}}\right\}^{1 / 2} .
$$

Proof. In view of (4.4), we use

$$
\begin{aligned}
& \left|\frac{z}{\sqrt{x+y}}-\frac{z^{\prime}}{\sqrt{x^{\prime}+y^{\prime}}}\right| \\
& \quad \leq \frac{\left|z-z^{\prime}\right|}{\sqrt{x^{\prime}}}+|z| \frac{\left|\sqrt{x}-\sqrt{x^{\prime}}\right|}{\sqrt{x} \sqrt{x^{\prime}}}+|z| \frac{\left|y-y^{\prime}\right|}{\sqrt{x} x^{\prime}} \quad\left(x, y, x^{\prime}, y^{\prime}>0, z, z^{\prime} \in \mathbb{R}\right)
\end{aligned}
$$

to obtain

$$
\begin{aligned}
& \sqrt{2}\left|\frac{\tilde{b}_{\lambda}(\vartheta)}{\sqrt{\tilde{a}_{\lambda}^{2}(\vartheta)+\tilde{b}_{\lambda}^{2}(\vartheta)}}-\frac{\lambda c_{2}|\vartheta|^{\alpha}(\vartheta /|\vartheta|)}{\sqrt{2} Q_{\lambda}\left(|\vartheta|^{\alpha}\right)}\right| \\
& \quad=\left|\frac{\lambda D(\vartheta)}{\sqrt{K_{\lambda}(\vartheta)+(1-\lambda+\lambda B(\vartheta)) K_{\lambda}(\vartheta)^{1 / 2}}}-\frac{\lambda c_{2}|\vartheta|^{\alpha}(\vartheta /|\vartheta|)}{\sqrt{k_{\lambda}(\vartheta)+\left(1-\lambda+\lambda c_{1}|\vartheta|^{\alpha}\right) k_{\lambda}(\vartheta)^{1 / 2}}}\right|
\end{aligned}
$$




$$
\begin{aligned}
\leq & \frac{\left.\left|D(\vartheta)-c_{2}\right| \vartheta\right|^{\alpha}(\vartheta /|\vartheta|) \mid}{k_{\lambda}(\vartheta)^{1 / 2}}+|D(\vartheta)| \frac{\left|K_{\lambda}(\vartheta)^{1 / 2}-k_{\lambda}(\vartheta)^{1 / 2}\right|}{K_{\lambda}(\vartheta)^{1 / 2} k_{\lambda}(\vartheta)^{1 / 2}} \\
& +|D(\vartheta)| \frac{\left|(1-\lambda+\lambda B(\vartheta)) K_{\lambda}(\vartheta)^{1 / 2}-\left(1-\lambda+\lambda c_{1}|\vartheta|^{\alpha}\right) k_{\lambda}(\vartheta)^{1 / 2}\right|}{K_{\lambda}(\vartheta)^{1 / 2} k_{\lambda}(\vartheta)} .
\end{aligned}
$$

From (A1) and

$$
k_{\lambda}(\vartheta)^{1 / 2} \geq \frac{1}{2} c_{1}|\vartheta|^{\alpha} \quad(\lambda \in(1 / 2,1), \vartheta \in[-\pi, \pi]),
$$

the first term on the right-hand side of the last inequality in (4.6) is bounded by a constant multiple of $|\vartheta|^{\varepsilon}$.

If $|\vartheta|$ is small enough (i.e. $c_{8}|\vartheta|^{\varepsilon} \leq 1 / 2$ ), then (4.5) gives

$$
K_{\lambda}(\vartheta)^{1 / 2} \geq \frac{1}{2} k_{\lambda}(\vartheta)^{1 / 2} .
$$

With the help of the above inequality and (4.7), (A1) and (4.5) imply that the second term on the right-hand side of the last inequality in (4.6) is bounded by a constant multiple of $|\vartheta|^{\varepsilon}$.

By using the triangle inequality, the third term on the right-hand side of the last inequality in (4.6) is bounded by

$$
|D(\vartheta)| \frac{\left.\left|B(\vartheta)-c_{1}\right| \vartheta\right|^{\alpha} \mid}{k_{\lambda}(\vartheta)}+\left.|D(\vartheta)| \frac{\left|K_{\lambda}(\vartheta)^{1 / 2}-k_{\lambda}(\vartheta)^{1 / 2}\right|}{K_{\lambda}(\vartheta)^{1 / 2} k_{\lambda}(\vartheta)}\left|1-\lambda+\lambda c_{1}\right| \theta\right|^{\alpha} \mid .
$$

From (A1) and (4.7), the first term of the above is bounded by a constant multiple of $|\vartheta|^{\varepsilon}$. It is proved that the second term of the above is bounded by a constant multiple of $|\vartheta|^{\varepsilon}$ by the same argument as showing that the second term on the right-hand side of the last inequality in (4.6) is bounded by a constant multiple of $|\vartheta|^{\varepsilon}$.

Hence, we have the desired inequality.

From (3.9) and the definitions of $A$ and $B$,

$$
\frac{1}{4} c_{*}\left|\theta_{2}\right|^{2} \leq \Re\left[\varphi_{\lambda}\left(\theta_{1}, \theta_{2}\right)\right]=\frac{\lambda}{2} E\left[X_{2}^{2}\right]\left\{\left(\theta_{2}+\frac{A}{2}\right)^{2}+B-\frac{A^{2}}{4}\right\}
$$

for $\lambda \in(1 / 2,1)$ and $\left|\left(\theta_{1}, \theta_{2}\right)\right| \leq r_{0}$. Since $A$ tends to zero as $\left|\theta_{1}\right| \rightarrow 0$, we choose $\theta_{1}$ small enough so that $\left|\left(\theta_{1},-A / 2\right)\right| \leq r_{0}$. Then the above inequality (setting $\theta_{2}=-A / 2$ ) gives

$$
\frac{c_{*}}{2 \lambda E\left[X_{2}^{2}\right]} A^{2} \leq 4 B-A^{2},
$$

and letting $\lambda \uparrow 1$,

$$
\frac{c_{*}}{2 E\left[X_{2}^{2}\right]} A^{2} \leq 4 \frac{1-E\left[\cos \theta_{1} X_{1}\right]}{(1 / 2) E\left[X_{2}^{2}\right]}-A^{2} \leq 8 \frac{B\left(\theta_{1}\right)}{E\left[X_{2}^{2}\right]} .
$$

From $1-E\left[\cos \theta_{1} X_{1}\right] \geq\left(E\left[\left(\sin \theta_{1} X_{1}\right) X_{2}\right]\right)^{2} /\left(2 E\left[X_{2}^{2}\right]\right)$, it follows that

$$
C^{2} \leq \frac{8}{E\left[X_{2}^{2}\right]} B\left(\theta_{1}\right)
$$

Combining the above inequalities with $2|D| \leq|2 D-A C|+|A||C|$, and using (4.5), Lemmas 3.2-3.5 give the following lemma. 
LEMmA 4.3. Assume that $(a)-(d)$ and (A1) hold. Then there exist $c_{10}>0, \lambda_{*} \in(1 / 2,1)$ and $s_{*} \in(0,1)$ such that, for $\lambda \in\left(\lambda_{*}, 1\right)$ and $0<|\vartheta| \leq s_{*}$,

$$
\begin{gathered}
\left|a_{\lambda}(\vartheta)-\tilde{a}_{\lambda}(\vartheta)\right| \leq c_{10}\left(|\vartheta|^{\hat{\delta}}+k_{\lambda}^{\delta / 4}(\vartheta)\left|\log k_{\lambda}(\vartheta)\right|\right) k_{\lambda}^{-1 / 4}(\vartheta), \\
\left|b_{\lambda}(\vartheta)-\tilde{b}_{\lambda}(\vartheta)\right| \leq c_{10}|\vartheta|^{\tilde{\delta}} k_{\lambda}^{-1 / 4}(\vartheta), \\
\left|\frac{b_{\lambda}(\vartheta)}{\sqrt{a_{\lambda}^{2}(\vartheta)+b_{\lambda}^{2}(\vartheta)}}-\frac{\tilde{b}_{\lambda}(\vartheta)}{\sqrt{\tilde{a}_{\lambda}^{2}(\vartheta)+\tilde{b}_{\lambda}^{2}(\vartheta)}}\right| \leq c_{10}|\vartheta|^{\tilde{\delta}}\left|\log k_{\lambda}(\vartheta)\right|,
\end{gathered}
$$

where $\tilde{\delta}=\min \{\hat{\delta}, \alpha \delta / 2\}$.

LEMmA 4.4. Assume that $(a)-(d)$ and (A1) hold. Then there exist $c_{11}>0, \lambda^{*} \in(1 / 2,1)$ and $s^{*} \in(0,1)$ such that, for $\lambda \in\left(\lambda^{*}, 1\right)$ and $0<|\vartheta| \leq s^{*}$,

$$
\left|\frac{b_{\lambda}(\vartheta)}{\sqrt{a_{\lambda}^{2}(\vartheta)+b_{\lambda}^{2}(\vartheta)}}-\frac{\lambda c_{2}|\vartheta|^{\alpha}(\vartheta /|\vartheta|)}{\sqrt{2} Q_{\lambda}\left(|\vartheta|^{\alpha}\right)}\right| \leq c_{11}|\vartheta|^{\delta_{0}},
$$

where $\delta_{0}=\min \{\varepsilon, \tilde{\delta} / 2\}$.

Proof. We apply Lemmas 4.2 and 4.3 to obtain

$$
\left|\frac{b_{\lambda}(\vartheta)}{\sqrt{a_{\lambda}^{2}(\vartheta)+b_{\lambda}^{2}(\vartheta)}}-\frac{\lambda c_{2}|\vartheta|^{\alpha}(\vartheta /|\vartheta|)}{\sqrt{2} Q_{\lambda}\left(|\vartheta|^{\alpha}\right)}\right| \leq c_{9}|\vartheta|^{\varepsilon}+c_{10}|\vartheta|^{\tilde{\delta}}\left|\log k_{\lambda}(\vartheta)\right| .
$$

From the definition of $k_{\lambda}(\vartheta)$, it is easy to see that

$$
\frac{1}{4}\left(c_{1}^{2}+c_{2}^{2}\right)|\theta|^{2 \alpha} \leq k_{\lambda}(\vartheta) \leq 1 \quad\left(\lambda \in(1 / 2,1),|\vartheta|^{\alpha} \leq 1 /\left(4 \max \left\{c_{1}, c_{2}\right\}\right)\right),
$$

which gives

$$
|\vartheta|^{\tilde{\delta} / 2}\left|\log k_{\lambda}(\vartheta)\right| \leq\left.|\vartheta|^{\tilde{\delta} / 2}\left|\log \frac{1}{4}\left(c_{1}^{2}+c_{2}^{2}\right)\right| \theta\right|^{2 \alpha} \mid .
$$

If $\vartheta$ is small enough, then the right-hand side of the above inequality is bounded by 1 and the second term of the right-hand side of (4.9) is bounded by $c_{10}|\theta|^{\tilde{\delta} / 2}$. Thus, we have (4.8).

LEMmA 4.5. Assume that $(a)-(d)$ and (Al) hold. Then there exist $c_{12}>0, \lambda_{0} \in(1 / 2,1)$ and $s_{0} \in(0,1)$ such that, for $\lambda \in\left(\lambda_{0}, 1\right)$ and $0<|\vartheta| \leq s_{0}$,

$$
\left|\arcsin \frac{b_{\lambda}(\vartheta)}{\sqrt{a_{\lambda}^{2}(\vartheta)+b_{\lambda}^{2}(\vartheta)}}-\arcsin \frac{\lambda c_{2}|\vartheta|^{\alpha}(\vartheta /|\vartheta|)}{\sqrt{2} Q_{\lambda}\left(|\vartheta|^{\alpha}\right)}\right| \leq c_{12}|\vartheta|^{\delta_{0}}
$$

Proof. By applying the mean value theorem to the inverse of the sin function, arcsin $t$, on the closed interval with end points $\lambda c_{2}|\vartheta|^{\alpha}(\vartheta /|\vartheta|) /\left(\sqrt{2} Q_{\lambda}\left(|\vartheta|^{\alpha}\right)\right)$ and $b_{\lambda}(\vartheta) / \sqrt{a_{\lambda}^{2}(\vartheta)+b_{\lambda}^{2}(\vartheta)}$, the left-hand side of (4.10) is equal to

$$
\frac{1}{\sqrt{1-t_{0}^{2}}}\left|\frac{b_{\lambda}(\vartheta)}{\sqrt{a_{\lambda}^{2}(\vartheta)+b_{\lambda}^{2}(\vartheta)}}-\frac{\lambda c_{2}|\vartheta|^{\alpha}(\vartheta /|\vartheta|)}{\sqrt{2} Q_{\lambda}\left(|\vartheta|^{\alpha}\right)}\right|,
$$

where $t_{0}$ is a number between $\lambda c_{2}|\vartheta|^{\alpha}(\vartheta /|\vartheta|) /\left(\sqrt{2} Q_{\lambda}\left(|\vartheta|^{\alpha}\right)\right)$ and $b_{\lambda}(\vartheta) / \sqrt{a_{\lambda}^{2}(\vartheta)+b_{\lambda}^{2}(\vartheta)}$. 
Note that for $\vartheta \neq 0$,

$$
\left|\frac{\lambda c_{2}|\vartheta|^{\alpha}(\vartheta /|\vartheta|)}{\sqrt{2} Q_{\lambda}\left(|\vartheta|^{\alpha}\right)}\right| \leq \frac{1}{\sqrt{2}}
$$

If $\vartheta$ is small enough, then (4.8) implies that

$$
\left|\frac{b_{\lambda}(\vartheta)}{\sqrt{a_{\lambda}^{2}(\vartheta)+b_{\lambda}^{2}(\vartheta)}}\right| \leq \frac{1}{\sqrt{2}}+c_{11}|\vartheta|^{\delta_{0}}<\frac{1}{2}\left(1+\frac{1}{\sqrt{2}}\right)
$$

and we obtain

$$
\left|t_{0}\right| \leq \max \left\{\left|\frac{\lambda c_{2}|\vartheta|^{\alpha}(\vartheta /|\vartheta|)}{\sqrt{2} Q_{\lambda}\left(|\vartheta|^{\alpha}\right)}\right|,\left|\frac{b_{\lambda}(\vartheta)}{\sqrt{a_{\lambda}^{2}(\vartheta)+b_{\lambda}^{2}(\vartheta)}}\right|\right\} \leq \frac{1}{2}\left(1+\frac{1}{\sqrt{2}}\right)<1 .
$$

Equation (4.11), together with (4.8) and the above inequality, gives the desired estimate (4.10).

To estimate $\lim _{L \rightarrow \infty} C_{L}(\lambda)$, we decompose $C_{L}(\lambda)$. With $s_{0}>0$ being the same constant as in Lemma 4.5,

$$
\begin{aligned}
C_{L}(\lambda)= & \frac{1}{\pi} \int_{-s_{0}}^{s_{0}} \frac{1}{\vartheta}\left(\arcsin \frac{\lambda c_{2}|\vartheta|^{\alpha}(\vartheta /|\vartheta|)}{\sqrt{2} Q_{\lambda}\left(|\vartheta|^{\alpha}\right)}\right) d \vartheta \\
& +I_{1}\left(s_{0}, \lambda\right)+I_{2}(\lambda, L)+I_{3}(\lambda, L)+I_{4}\left(s_{0}, \lambda\right),
\end{aligned}
$$

where

$$
\begin{aligned}
I_{1}\left(s_{0}, \lambda\right)= & \frac{1}{2 \pi} \int_{-s_{0}}^{s_{0}} \frac{\sin \vartheta}{1-\cos \vartheta}\left(\arcsin \frac{b_{\lambda}(\vartheta)}{\sqrt{a_{\lambda}^{2}(\vartheta)+b_{\lambda}^{2}(\vartheta)}}\right) \\
& -\frac{2}{\vartheta}\left(\arcsin \frac{\lambda c_{2}|\vartheta|^{\alpha}(\vartheta /|\vartheta|)}{\sqrt{2} Q_{\lambda}\left(|\vartheta|^{\alpha}\right)}\right) d \vartheta \\
I_{2}(\lambda, L)= & \frac{1}{2 \pi} \int_{-\pi}^{\pi}\left(\arcsin \frac{b_{\lambda}(\vartheta)}{\sqrt{a_{\lambda}^{2}(\vartheta)+b_{\lambda}^{2}(\vartheta)}}\right) \sin (L \vartheta) d \vartheta \\
I_{3}(\lambda, L)= & -\frac{1}{2 \pi} \int_{-\pi}^{\pi}\left(\arcsin \frac{b_{\lambda}(\vartheta)}{\sqrt{a_{\lambda}^{2}(\vartheta)+b_{\lambda}^{2}(\vartheta)}}\right) \frac{\sin \vartheta}{1-\cos \vartheta} \cos (L \vartheta) d \vartheta \\
I_{4}\left(s_{0}, \lambda\right)= & \frac{1}{2 \pi} \int_{s_{0}<|\vartheta| \leq \pi} \frac{\sin \vartheta}{1-\cos \vartheta}\left(\arcsin \frac{b_{\lambda}(\vartheta)}{\sqrt{a_{\lambda}^{2}(\vartheta)+b_{\lambda}^{2}(\vartheta)}}\right) d \vartheta
\end{aligned}
$$

Since $\arcsin t \in[-\pi / 2, \pi / 2], \arcsin \left(b_{\lambda}(\vartheta) / \sqrt{a_{\lambda}^{2}(\vartheta)+b_{\lambda}^{2}(\vartheta)}\right)$ is integrable on $[-\pi, \pi]$. The Riemann-Lebesgue lemma implies that

$$
\lim _{L \rightarrow \infty} I_{2}(\lambda, L)=0 .
$$

It is easy to verify that

$$
\begin{aligned}
\left|I_{4}\left(s_{0}, \lambda\right)\right| & \leq \frac{1}{2 \pi} \int_{s_{0}<|\vartheta| \leq \pi}\left|\frac{\sin \vartheta}{1-\cos \vartheta}\left(\arcsin \frac{b_{\lambda}(\vartheta)}{\sqrt{a_{\lambda}^{2}(\vartheta)+b_{\lambda}^{2}(\vartheta)}}\right)\right| d \vartheta \\
& \leq \frac{\pi}{2} \frac{1}{1-\cos s_{0}} .
\end{aligned}
$$


By applying mean value theorem to the function arcsin $t$ on the closed interval with end points $\lambda c_{2}|\vartheta|^{\alpha}(\vartheta /|\vartheta|) /\left(\sqrt{2} Q_{\lambda}\left(|\vartheta|^{\alpha}\right)\right)$ and 0 , and using (4.12),

$$
\left|\arcsin \frac{\lambda c_{2}|\vartheta|^{\alpha}(\vartheta /|\vartheta|)}{\sqrt{2} Q_{\lambda}\left(|\vartheta|^{\alpha}\right)}\right| \leq \sqrt{2}\left|\frac{\lambda c_{2}|\vartheta|^{\alpha}(\vartheta /|\vartheta|)}{\sqrt{2} Q_{\lambda}\left(|\vartheta|^{\alpha}\right)}\right| \leq \frac{\lambda c_{2}|\vartheta|^{\alpha}}{1-\lambda}
$$

This inequality and (4.10) give

$$
\left|\left(\arcsin \frac{b_{\lambda}(\vartheta)}{\sqrt{a_{\lambda}^{2}(\vartheta)+b_{\lambda}^{2}(\vartheta)}}\right) \frac{\sin \vartheta}{1-\cos \vartheta}\right| \leq \begin{cases}\left(\frac{\lambda c_{2}|\vartheta|^{\alpha}}{1-\lambda}+c_{12}|\vartheta|^{\delta_{0}}\right) \frac{\pi}{|\vartheta|} & \left(0<|\vartheta| \leq s_{0}\right), \\ \frac{\pi}{2} \frac{1}{1-\cos s_{0}} & \left(s_{0}<|\vartheta| \leq \pi\right) .\end{cases}
$$

For fixed $\lambda \in\left(\lambda_{0}, 1\right)$, the above inequality implies that the left-hand side of the above is integrable on $[-\pi, \pi]$. By the Riemann-Lebesgue lemma,

$$
\lim _{L \rightarrow \infty} I_{3}(\lambda, L)=0 .
$$

As for $I_{1}\left(s_{0}, \lambda\right)$, we consider the absolute value of the integrand in $I_{1}\left(s_{0}, \lambda\right)$. From (4.10) and $\arcsin t \in[-\pi / 2, \pi / 2]$,

$$
\begin{aligned}
& \left|\frac{\sin \vartheta}{1-\cos \vartheta}\left(\arcsin \frac{b_{\lambda}(\vartheta)}{\sqrt{a_{\lambda}^{2}(\vartheta)+b_{\lambda}^{2}(\vartheta)}}\right)-\frac{2}{\vartheta}\left(\arcsin \frac{\lambda c_{2}|\vartheta|^{\alpha}(\vartheta /|\vartheta|)}{\sqrt{2} Q_{\lambda}\left(|\vartheta|^{\alpha}\right)}\right)\right| \\
& \leq \frac{2}{|\vartheta|} c_{12}|\vartheta|^{\delta_{0}}+\left|\frac{\sin \vartheta}{1-\cos \vartheta}-\frac{2}{\vartheta}\right| \frac{\pi}{2} \leq 2 c_{12}|\vartheta|^{\delta_{0}-1}+\frac{\pi}{2}|\vartheta| .
\end{aligned}
$$

Hence, $I_{1}\left(s_{0}, \lambda\right)$ is bounded by a constant independent of $\lambda \in\left(\lambda_{0}, 1\right)$. We have the following estimate.

There exists a constant $c_{13}$ such that, for $\lambda \in\left(\lambda_{0}, 1\right)$,

$$
\left|\lim _{L \rightarrow \infty} C_{L}(\lambda)-\frac{1}{\pi} \int_{-s_{0}}^{s_{0}} \frac{1}{\vartheta}\left(\arcsin \frac{\lambda c_{2}|\vartheta|^{\alpha}(\vartheta /|\vartheta|)}{\sqrt{2} Q_{\lambda}\left(|\vartheta|^{\alpha}\right)}\right) d \vartheta\right| \leq c_{13} .
$$

Lemma 4.6. Assume that $(a)-(d)$ and (Al) hold. Then there exists a constant $c_{14}>0$ such that, for $\lambda \in\left(\lambda_{0}, 1\right)$,

$$
\left|\lim _{L \rightarrow \infty} C_{L}(\lambda)+\frac{2}{\alpha \pi}\left(\arcsin \frac{c_{2}}{\sqrt{2} \sqrt{c_{1}^{2}+c_{2}^{2}+c_{1} \sqrt{c_{1}^{2}+c_{2}^{2}}}}\right) \log (1-\lambda)\right| \leq c_{14} .
$$

Proof. Equation (4.13) reduces our problem to the estimate for

$$
I_{0}\left(s_{0}, \lambda\right):=\frac{1}{\pi} \int_{-s_{0}}^{s_{0}} \frac{1}{\vartheta}\left(\arcsin \frac{\lambda c_{2}|\vartheta|^{\alpha}(\vartheta /|\vartheta|)}{\sqrt{2} Q_{\lambda}\left(|\vartheta|^{\alpha}\right)}\right) d \vartheta
$$

To estimate this integral, we use the power series representation for $\arcsin t$ :

$$
\arcsin t=\sum_{n=0}^{\infty} \frac{1 \cdot 3 \cdot 5 \cdots(2 n-1)}{2 \cdot 4 \cdot 6 \cdots 2 n} \frac{t^{2 n+1}}{2 n+1} \quad(-1<t<1) .
$$

By the dominated convergence theorem,

$$
I_{0}\left(s_{0}, \lambda\right)=\sum_{n=0}^{\infty} \frac{1 \cdot 3 \cdot 5 \cdots(2 n-1)}{2 \cdot 4 \cdot 6 \cdots 2 n} \frac{1}{2 n+1} \frac{1}{\pi} \int_{-s_{0}}^{s_{0}} \frac{1}{\vartheta}\left(\frac{\lambda c_{2}|\vartheta|^{\alpha}(\vartheta /|\vartheta|)}{\sqrt{2} Q_{\lambda}\left(|\vartheta|^{\alpha}\right)}\right)^{2 n+1} d \vartheta
$$


We note that the function

$$
\frac{1}{\vartheta}\left(\frac{\lambda c_{2}|\vartheta|^{\alpha}(\vartheta /|\vartheta|)}{\sqrt{2} Q_{\lambda}\left(|\vartheta|^{\alpha}\right)}\right)^{2 n+1}
$$

(on the right-hand side of (4.15)) is an even function, and perform the change of variables $s=\vartheta^{\alpha}$, so that

$$
\begin{aligned}
\int_{-s_{0}}^{s_{0}} \frac{1}{\vartheta}\left(\frac{\lambda c_{2}|\vartheta|^{\alpha}(\vartheta /|\vartheta|)}{\sqrt{2} Q_{\lambda}\left(|\vartheta|^{\alpha}\right)}\right)^{2 n+1} d \vartheta & =2 \int_{0}^{s_{0}} \frac{1}{\vartheta}\left(\frac{\lambda c_{2}|\vartheta|^{\alpha}}{\sqrt{2} Q_{\lambda}\left(|\vartheta|^{\alpha}\right)}\right)^{2 n+1} d \vartheta \\
& =\frac{2}{\alpha}\left(\frac{\lambda c_{2}}{\sqrt{2}}\right)^{2 n+1} \int_{0}^{s_{0}^{\alpha}} \frac{s^{2 n}}{\left(Q_{\lambda}(s)\right)^{2 n+1}} d s .
\end{aligned}
$$

Put

$$
Q_{\lambda}^{(1)}(s)=\left\{\lambda^{2}\left(c_{1}^{2}+c_{2}^{2}+c_{1} \sqrt{c_{1}^{2}+c_{2}^{2}}\right) s^{2}+(1-\lambda) \lambda\left(3 c_{1}+\sqrt{c_{1}^{2}+c_{2}^{2}}\right) s+2(1-\lambda)^{2}\right\}^{1 / 2}
$$

and

$$
\begin{aligned}
Q_{\lambda}^{(2)}(s)= & \left\{\lambda^{2}\left(c_{1}^{2}+c_{2}^{2}+c_{1} \sqrt{c_{1}^{2}+c_{2}^{2}}\right) s^{2}\right. \\
& \left.+(1-\lambda) \lambda\left(2 c_{1}+\sqrt{c_{1}^{2}+c_{2}^{2}}+\frac{c_{1}^{2}}{\sqrt{c_{1}^{2}+c_{2}^{2}}}\right) s+(1-\lambda)^{2}\left(1+\frac{c_{1}}{\sqrt{c_{1}^{2}+c_{2}^{2}}}\right)\right\}^{1 / 2} .
\end{aligned}
$$

Here $Q_{\lambda}^{(1)}(s)$ is obtained by replacing $\sqrt{\left(1-\lambda+\lambda c_{1} s\right)^{2}+\left(\lambda c_{2} s\right)^{2}}$ in the definition of $Q_{\lambda}(s)$ with $\lambda \sqrt{c_{1}^{2}+c_{2}^{2}} s+1-\lambda$ and $Q_{\lambda}^{(2)}(s)$ is obtained by replacing $\sqrt{\left(1-\lambda+\lambda c_{1} s\right)^{2}+\left(\lambda c_{2} s\right)^{2}}$ in the definition of $Q_{\lambda}(s)$ with $\lambda \sqrt{c_{1}^{2}+c_{2}^{2}} s+(1-\lambda)\left(c_{1} / \sqrt{c_{1}^{2}+c_{2}^{2}}\right)$.

From

$$
\lambda \sqrt{c_{1}^{2}+c_{2}^{2}} s+(1-\lambda) \frac{c_{1}}{\sqrt{c_{1}^{2}+c_{2}^{2}}} \leq \sqrt{\left(1-\lambda+\lambda c_{1} s\right)^{2}+\left(\lambda c_{2} s\right)^{2}} \leq \lambda \sqrt{c_{1}^{2}+c_{2}^{2}} s+1-\lambda
$$

for $s>0$, we have

$$
\int_{0}^{s_{0}^{\alpha}} \frac{s^{2 n}}{\left(Q_{\lambda}^{(1)}(s)\right)^{2 n+1}} d s \leq \int_{0}^{s_{0}^{\alpha}} \frac{s^{2 n}}{\left(Q_{\lambda}(s)\right)^{2 n+1}} d s \leq \int_{0}^{s_{0}^{\alpha}} \frac{s^{2 n}}{\left(Q_{\lambda}^{(2)}(s)\right)^{2 n+1}} d s .
$$

For $a, b, c \in(0, \infty)$ with $4 a c-b^{2}>0$, we next evaluate the integral

$$
I_{n}^{\alpha}\left(s_{0}\right):=\int_{0}^{s_{0}^{\alpha}} \frac{s^{2 n}}{\left(a s^{2}+b s+c\right)^{(2 n+1) / 2}} d s \quad(n \in\{0\} \cup \mathbb{N}) .
$$

If $n=0$,

$$
I_{0}^{\alpha}\left(s_{0}\right)=\frac{1}{\sqrt{a}}\left[\log \left(2 a s+b+2 \sqrt{a\left(a s^{2}+b s+c\right)}\right)\right]_{0}^{s_{0}^{\alpha}} .
$$

By setting $a=\lambda^{2}\left(c_{1}^{2}+c_{2}^{2}+c_{1} \sqrt{c_{1}^{2}+c_{2}^{2}}\right), b=(1-\lambda) \lambda\left(3 c_{1}+\sqrt{c_{1}^{2}+c_{2}^{2}}\right), c=2(1-\lambda)^{2}$, this identity yields the following estimate. 
There exists a constant $c_{15}>0$ such that, for $\lambda \in(1 / 2,1)$,

$$
\left|\frac{\lambda c_{2}}{\sqrt{2}} \int_{0}^{s_{0}^{\alpha}} \frac{1}{Q_{\lambda}^{(1)}(s)} d s+\frac{c_{2}}{\sqrt{2} \sqrt{c_{1}^{2}+c_{2}^{2}+c_{1} \sqrt{c_{1}^{2}+c_{2}^{2}}}} \log (1-\lambda)\right| \leq c_{15} .
$$

If $n \geq 1$, we note that

$$
\frac{d}{d s} \frac{1}{\left(a s^{2}+b s+c\right)^{(2 n-1) / 2}}=-\frac{2 n-1}{2} \frac{2 a s+b}{\left(a s^{2}+b s+c\right)^{(2 n+1) / 2}} .
$$

The formula for integration by parts gives

$$
I_{n}^{\alpha}\left(s_{0}\right)=\frac{1}{a} I_{n-1}^{\alpha}\left(s_{0}\right)+e_{n},
$$

where

$$
e_{n}=-\frac{1}{(2 n-1) a} \frac{s_{0}^{(2 n-1) \alpha}}{\left(a s_{0}^{2 \alpha}+b s_{0}^{\alpha}+c\right)^{(2 n-1) / 2}}-\frac{b}{2 a} \int_{0}^{s_{0}^{\alpha}} \frac{s^{2 n-1}}{\left(a s^{2}+b s+c\right)^{(2 n+1) / 2}} d s .
$$

By using the above relation repeatedly, we obtain

$$
I_{n}^{\alpha}\left(s_{0}\right)=\frac{1}{a^{n}} I_{0}^{\alpha}\left(s_{0}\right)+\sum_{j=0}^{n-1} \frac{1}{a^{j}} e_{n-j} .
$$

Since $a, b, c$ are positive and satisfy the inequality $4 a c-b^{2}>0$,

$$
\begin{aligned}
\left|e_{n}\right| & \leq \frac{1}{a^{(2 n+1) / 2}}+\frac{b}{2 a^{(2 n+1) / 2}} \int_{0}^{s_{0}^{\alpha}} \frac{1}{a s^{2}+b s+c} d s \\
& \leq \frac{1}{a^{(2 n+1) / 2}}\left(1+\frac{\pi}{2} \frac{b}{\sqrt{4 a c-b^{2}}}\right) .
\end{aligned}
$$

With the help of (4.19) and (4.20), and by setting $a=\lambda^{2}\left(c_{1}^{2}+c_{2}^{2}+c_{1} \sqrt{c_{1}^{2}+c_{2}^{2}}\right), b=$ $(1-\lambda) \lambda\left(3 c_{1}+\sqrt{c_{1}^{2}+c_{2}^{2}}\right), c=2(1-\lambda)^{2},(4.18)$ implies the following estimate for $k=1$.

There exists a constant $c_{16}>0$ such that, for $\lambda \in(1 / 2,1)$ and $n \in\{0\} \cup \mathbb{N}$,

$$
\begin{aligned}
& \left|\left(\frac{\lambda c_{2}}{\sqrt{2}}\right)^{2 n+1} \int_{0}^{s_{0}^{\alpha}} \frac{s^{2 n}}{\left(Q_{\lambda}^{(k)}(s)\right)^{2 n+1}} d s+\left(\frac{c_{2}}{\sqrt{2} \sqrt{c_{1}^{2}+c_{2}^{2}+c_{1} \sqrt{c_{1}^{2}+c_{2}^{2}}}}\right)^{2 n+1} \log (1-\lambda)\right| \\
& \quad \leq c_{16}(n+1)\left(\frac{1}{\sqrt{2}}\right)^{2 n}(k=1,2)
\end{aligned}
$$

The same argument as in the proof of the estimate for $k=1$ in (4.21) gives the estimate for $k=2$ in (4.21).

By (4.17), (4.21) also holds when the integral of $s^{2 n} /\left(Q_{\lambda}^{(k)}(s)\right)^{2 n+1}$ over $\left[0, s_{0}^{\alpha}\right]$ with respect to $s$ is replaced by the integral of $s^{2 n} /\left(Q_{\lambda}(s)\right)^{2 n+1}$ over $\left[0, s_{0}^{\alpha}\right]$ with respect to $s$. This estimate implies (4.14) in view of (4.16) and (4.15).

We recall (4.1). It immediate from Lemma 4.6 that, for $\lambda \in\left(\lambda_{0}, 1\right)$,

$$
(1-\lambda)^{-2 \beta} e^{-c_{14}} \leq \frac{P_{0}\left\{T_{\lambda}<\tau_{V_{-}}\right\}}{P_{0}\left\{T_{\lambda}<\tau_{V_{+}}\right\}} \leq(1-\lambda)^{-2 \beta} e^{c_{14}},
$$


where $\beta$ is the same constant as in Theorem 1.1. By combining the above inequalities, Lemmas 2.1 and 2.2, we have the following estimate.

Proposition 4.7. Assume that $(a)-(d)$ and (A1) hold. Then there exist constants $c_{17}, c_{18}>$ 0 and $\lambda_{0}^{*} \in(1 / 2,1)$ such that, for $\lambda \in\left(\lambda_{0}^{*}, 1\right)$,

$$
c_{17}(1-\lambda)^{-\beta+(1 / 4)} \leq P_{0}\left\{T_{\lambda}<\tau_{V_{-}}\right\}=(1-\lambda) \sum_{n=0}^{\infty} \lambda^{n} P_{0}\left\{\tau_{V_{-}}>n\right\} \leq c_{18}(1-\lambda)^{-\beta+(1 / 4)} .
$$

This asymptotic behavior of $P_{0}\left\{T_{\lambda}<\tau_{V_{-}}\right\}$implies (1.3) by the Tauberian theorem (see [5, Theorem 2.4.3]). Thus, we have (1.3) in Theorem 1.1 for $c_{1}>0$ and $c_{2}>0$.

The other proofs are similar. The proof of Theorem 1.1 is complete.

\section{A. Appendix: Proof of Lemmas 3.2-3.5}

We calculate

$$
\Re\left[\frac{1}{2 \pi} \int_{-r_{0} / 2}^{r_{0} / 2} \frac{1}{\varphi_{\lambda}(\theta)} d \theta_{2}\right]=\frac{1}{2 \pi} \int_{-r_{0} / 2}^{r_{0} / 2} \Re\left[\frac{1}{\varphi_{\lambda}(\theta)}\right] d \theta_{2},
$$

where $r_{0}$ is the constant in Lemma 3.1.

Recall that

$$
\begin{gathered}
A=\frac{E\left[\left(\sin \theta_{1} X_{1}\right) X_{2}\right]}{(1 / 2) E\left[X_{2}^{2}\right]}, \quad B=\frac{1-\lambda E\left[\cos \theta_{1} X_{1}\right]}{(\lambda / 2) E\left[X_{2}^{2}\right]}, \\
C=\frac{E\left[\left(-1+\cos \theta_{1} X_{1}\right) X_{2}\right]}{(1 / 2) E\left[X_{2}^{2}\right]}, \quad D=\frac{E\left[\sin \theta_{1} X_{1}\right]}{(1 / 2) E\left[X_{2}^{2}\right]}, \\
K=\left(4 B-A^{2}+C^{2}\right)^{2}+4(2 D-A C)^{2}
\end{gathered}
$$

and

$$
\varphi_{\lambda}(\theta)=\frac{\lambda}{2} E\left[X_{2}^{2}\right]\left\{\theta_{2}^{2}+A \theta_{2}+B-i\left(C \theta_{2}+D\right)\right\}
$$

Put

$$
\begin{gathered}
a_{ \pm}=A \pm \sqrt{H} \\
b_{ \pm}=\left\{\begin{array}{lc}
\frac{1}{4}\left\{A^{2}+C^{2}+\sqrt{K} \pm \frac{2 A H-2 C(2 D-A C)}{\sqrt{H}}\right\}, & 2 D-A C \neq 0 \\
\frac{1}{2}\left\{2 B+C^{2} \pm \sqrt{4 B-A^{2}+C^{2}}|C|\right\}, & 2 D-A C=0
\end{array}\right.
\end{gathered}
$$

where

$$
H=\frac{1}{2}\left(-4 B+A^{2}-C^{2}+\sqrt{K}\right) .
$$

Then $a_{ \pm}, b_{ \pm}$are real numbers, since $4 B-A^{2}-C^{2}>0$, and

$$
\left(\theta_{2}^{2}+A \theta_{2}+B\right)^{2}+\left(C \theta_{2}+D\right)^{2}=\left(\theta_{2}^{2}+a_{+} \theta_{2}+b_{+}\right)\left(\theta_{2}^{2}+a_{-} \theta_{2}+b_{-}\right) .
$$

In the case $2 D-A C \neq 0$, it is easy to verify that

$$
M:=\left(a_{+}-a_{-}\right)\left(a_{+} b_{-}-a_{-} b_{+}\right)+\left(b_{-}-b_{+}\right)^{2}=\frac{1}{2}\left(-4 B+A^{2}+C^{2}+\sqrt{K}\right) \sqrt{K} \neq 0
$$


using

$$
\left(b_{-}-b_{+}\right)^{2}=\left\{-\frac{A H-C(2 D-A C)}{\sqrt{H}}\right\}^{2}=\frac{1}{4}\left(A^{2}+C^{2}+\sqrt{K}\right)^{2}-4\left(B^{2}+D^{2}\right) \text {. }
$$

From the definition of $a_{ \pm}$and $b_{ \pm}$

$$
4 b_{ \pm}-a_{ \pm}^{2}=J_{1} \pm J_{2}
$$

where

$$
J_{1}=\frac{1}{2}\left(4 B-A^{2}+3 C^{2}+\sqrt{K}\right), \quad J_{2}=-\frac{2 C(2 D-A C)}{\sqrt{H}} .
$$

From (A.2), $4 B-A^{2}-C^{2}>0$ implies

$$
J_{1}^{2}-J_{2}^{2}=\frac{1}{4}\left(4 B-A^{2}-C^{2}+\sqrt{K}\right)^{2}>0 .
$$

Combining the above inequality with $J_{1}>0$, we have

$$
\left|J_{2}\right|<J_{1}
$$

and

$$
4 b_{ \pm}-a_{ \pm}^{2}>0
$$

Equation (A.4) means that $\theta_{2}^{2}+a_{ \pm} \theta_{2}+b_{ \pm}$is not zero for any $\theta_{2} \in \mathbb{R}$.

The partial-fraction decomposition of $\mathfrak{R}\left[1 / \varphi_{\lambda}(\theta)\right]$ is

$$
\Re\left[\frac{1}{\varphi_{\lambda}(\theta)}\right]=\frac{\Re\left[\varphi_{\lambda}(\theta)\right]}{\left|\varphi_{\lambda}(\theta)\right|^{2}}=\frac{1}{(\lambda / 2) E\left[X_{2}^{2}\right]}\left(\frac{F \theta_{2}+G}{\theta_{2}^{2}+a_{+} \theta_{2}+b_{+}}+\frac{-F \theta_{2}+I}{\theta_{2}^{2}+a_{-} \theta_{2}+b_{-}}\right),
$$

where

$$
\begin{aligned}
F & =\frac{-\left(a_{+} b_{-}-a_{-} b_{+}\right)+A\left(b_{-}-b_{+}\right)+B\left(a_{+}-a_{-}\right)}{M}, \\
G & =\frac{-b_{+}\left(b_{-}-b_{+}\right)-A\left(a_{+}-a_{-}\right) b_{+}+B a_{+}\left(a_{+}-a_{-}\right)+B\left(b_{-}-b_{+}\right)}{M}, \\
I & =\frac{b_{-}\left(b_{-}-b_{+}\right)+A\left(a_{+}-a_{-}\right) b_{-}-B a_{-}\left(a_{+}-a_{-}\right)-B\left(b_{-}-b_{+}\right)}{M} .
\end{aligned}
$$

We turn now to the integration. In the case $2 D-A C \neq 0$,

$$
\begin{aligned}
& \frac{\lambda E\left[X_{2}^{2}\right]}{2} \int_{-r_{0} / 2}^{r_{0} / 2} \Re\left[\frac{1}{\varphi_{\lambda}(\theta)}\right] d \theta_{2} \\
& =\int_{-r_{0} / 2}^{r_{0} / 2} \frac{F \theta_{2}+G}{\theta_{2}^{2}+a_{+} \theta_{2}+b_{+}}+\frac{-F \theta_{2}+I}{\theta_{2}^{2}+a_{-} \theta_{2}+b_{-}} d \theta_{2} \\
& =\frac{F}{2}\left[\log \frac{\theta_{2}^{2}+a_{+} \theta_{2}+b_{+}}{\theta_{2}^{2}+a_{-} \theta_{2}+b_{-}}\right]_{-r_{0} / 2}^{r_{0} / 2}+\frac{-a_{+} F+2 G}{\sqrt{4 b_{+}-a_{+}^{2}}}\left[\arctan \frac{2 \theta_{2}+a_{+}}{\sqrt{4 b_{+}-a_{+}^{2}}}\right]_{-r_{0} / 2}^{r_{0} / 2} \\
& \quad+\frac{a_{-} F+2 I}{\sqrt{4 b_{-}-a_{-}^{2}}}\left[\arctan \frac{2 \theta_{2}+a_{-}}{\sqrt{4 b_{-}-a_{-}^{2}}}\right]_{-r_{0} / 2}^{r_{0} / 2}
\end{aligned}
$$


In the case $2 D-A C=0$, we obtain

$$
\begin{aligned}
& \frac{\lambda E\left[X_{2}^{2}\right]}{2} \int_{-r_{0} / 2}^{r_{0} / 2} \Re\left[\frac{1}{\varphi_{\lambda}(\theta)}\right] d \theta_{2} \\
& =\frac{1}{\sqrt{4 B-A^{2}+C^{2}}}\left[\arctan \frac{2 \theta_{2}+A}{\sqrt{4 B-A^{2}+C^{2}}+|C|}\right]_{-r_{0} / 2}^{r_{0} / 2} \\
& \quad+\frac{1}{\sqrt{4 B-A^{2}+C^{2}}}\left[\arctan \frac{2 \theta_{2}+A}{\sqrt{4 B-A^{2}+C^{2}}-|C|}\right]_{-r_{0} / 2}^{r_{0} / 2}
\end{aligned}
$$

Proof of Lemma 3.2. We will prove (3.10) in the case $2 D-A C \neq 0$; the proof of (3.10) in the case $2 D-A C=0$ is similar and is omitted.

Assume that $2 D-A C \neq 0$. From the definition of $a_{ \pm}, a_{ \pm}$tends to 0 as $\theta_{1} \rightarrow 0$ and $\lambda \uparrow 1$. In view of (A.2) and the definition of $b_{ \pm}, b_{ \pm}$also tends to 0 as $\theta_{1} \rightarrow 0$ and $\lambda \uparrow 1$. If $a_{ \pm}$ and $b_{ \pm}$are small enough, then we can apply

$$
\log (1+x)|\leq 2| x \mid \quad(|x| \leq 1 / 2)
$$

to estimate the first term on the right-hand side of the last equality in (A.5) . With the help of

$$
F=-\frac{\sqrt{H}}{\sqrt{K}}
$$

the first term on the right-hand side of the last equality in (A.5) is bounded by a constant.

A simple calculation gives

$$
\begin{array}{r}
-a_{+} F+2 G=\frac{1}{2 \sqrt{K}}\left\{4 B-A^{2}+C^{2}+\sqrt{K}-\frac{2 C(2 D-A C)}{\sqrt{H}}\right\}, \\
a_{-} F+2 I=\frac{1}{2 \sqrt{K}}\left\{4 B-A^{2}+C^{2}+\sqrt{K}+\frac{2 C(2 D-A C)}{\sqrt{H}}\right\} .
\end{array}
$$

We note that

$$
\left|-\frac{2 C(2 D-A C)}{\sqrt{H}}\right|=\left|J_{2}\right|<J_{1}=\frac{1}{2}\left(4 B-A^{2}+3 C^{2}+\sqrt{K}\right),
$$

as shown in the proof of (A.4). Equation (A.6) implies that $\left|-a_{+} F+2 G\right| \leq 3 J_{1} /(2 \sqrt{K})$, which is bounded by a constant. The difference between the second term on the right-hand side of the last equality in (A.5) and

$$
\frac{-a_{+} F+2 G}{\sqrt{4 b_{+}-a_{+}^{2}}} \pi=\frac{\pi}{2 \sqrt{J_{1}+J_{2}} \sqrt{K}}\left\{4 B-A^{2}+C^{2}+\sqrt{K}-\frac{2 C(2 D-A C)}{\sqrt{H}}\right\}
$$

is bounded by a constant. Here we have used the inequality

$$
0 \leq \frac{\pi}{2}-\arctan x \leq \frac{1}{x} \quad(x>0) .
$$

Similarly, (A.7) implies that the difference between the third term on the right-hand side of the last equality in (A.5) and

$$
\frac{a_{-} F+2 I}{\sqrt{4 b_{-}-a_{-}^{2}}} \pi=\frac{\pi}{2 \sqrt{J_{1}-J_{2}} \sqrt{K}}\left\{4 B-A^{2}+C^{2}+\sqrt{K}+\frac{2 C(2 D-A C)}{\sqrt{H}}\right\}
$$


is bounded by a constant. Put

$$
\bar{a}_{\lambda}\left(\theta_{1}\right)=\frac{1}{2 \lambda E\left[X_{2}^{2}\right] \sqrt{K}}\left(\frac{J+J_{2}}{\sqrt{J_{1}+J_{2}}}+\frac{J-J_{2}}{\sqrt{J_{1}-J_{2}}}\right),
$$

where

$$
J=4 B-A^{2}+C^{2}+\sqrt{K} .
$$

Then the difference between $(1 / 2 \pi) \int_{-r_{0} / 2}^{r_{0} / 2} \mathfrak{R}\left[1 / \varphi_{\lambda}(\theta)\right] d \theta_{2}$ and $\bar{a}_{\lambda}\left(\theta_{1}\right)$ is bounded by a constant.

It remains to show that

$$
\bar{a}_{\lambda}\left(\theta_{1}\right)=\tilde{a}_{\lambda}\left(\theta_{1}\right):=\frac{\sqrt{2}}{\lambda E\left[X_{2}^{2}\right] \sqrt{K}} \sqrt{4 B-A^{2}+C^{2}+\sqrt{K}} .
$$

Since $4 B-A^{2}-C^{2}>0$,

$$
\left|J_{2}\right|<J_{1} \leq J
$$

which implies that $\bar{a}_{\lambda}\left(\theta_{1}\right) \geq 0$. (The first inequality above has already been shown in the proof of (A.4).) We will compute $\left(\bar{a}_{\lambda}\left(\theta_{1}\right)\right)^{2}$. By (A.3) and $J=2 J_{1}-2 C^{2}$,

$$
\begin{aligned}
\left\{\left(J+J_{2}\right) \sqrt{J_{1}-J_{2}}+\left(J-J_{2}\right) \sqrt{J_{1}+J_{2}}\right\}^{2} \\
=2\left(J_{1}+\sqrt{J_{1}^{2}-J_{2}^{2}}\right) J^{2}+2\left(J_{1}-2 J-\sqrt{J_{1}^{2}-J_{2}^{2}}\right) J_{2}^{2} \\
=8\left(4 B-A^{2}+C^{2}+\sqrt{K}\right)\left(J_{1}-C^{2}\right)^{2}-4\left(4 B-A^{2}+\sqrt{K}\right) J_{2}^{2} \\
=8\left(4 B-A^{2}+C^{2}+\sqrt{K}\right)\left(J_{1}^{2}-J_{2}^{2}\right)+8\left(4 B-A^{2}+C^{2}+\sqrt{K}\right)\left(-2 J_{1}+C^{2}\right) C^{2} \\
\quad+4\left(4 B-A^{2}+2 C^{2}+\sqrt{K}\right) J_{2}^{2} .
\end{aligned}
$$

In view of

$$
J_{2}^{2}=J_{1}^{2}-\frac{1}{4}\left(4 B-A^{2}-C^{2}+\sqrt{K}\right)^{2}=2\left(4 B-A^{2}+C^{2}+\sqrt{K}\right) C^{2},
$$

which follows from (A.3), the sum of the second and third terms on the right-hand side of the last equality in (A.9) vanish. Hence,

$$
\left(\bar{a}_{\lambda}\left(\theta_{1}\right)\right)^{2}=\left(\tilde{a}_{\lambda}\left(\theta_{1}\right)\right)^{2} .
$$

Since $\bar{a}_{\lambda}\left(\theta_{1}\right)$ and $\tilde{a}_{\lambda}\left(\theta_{1}\right)$ are non-negative functions, the above relation yields (A.8).

To show Lemma 3.3, we require the following lemmas.

Lemma A.1. Assume that $(a)-(d)$ hold. Then, for $\lambda \in(1 / 2,1)$ and $\theta_{1} \in[-\pi, \pi]$ with $\left|\theta_{1}\right| \leq r_{0} / 2$

(i) $\frac{1}{2 \pi} \int_{-r_{0} / 2}^{r_{0} / 2} \frac{\theta_{2}^{2}}{\left|\varphi_{\lambda}(\theta)\right|^{2}} d \theta_{2} \leq \frac{16}{c_{*} E\left[X_{2}^{2}\right]} K^{-1 / 4}$,

(ii) $\frac{1}{2 \pi} \int_{-r_{0} / 2}^{r_{0} / 2} \frac{1}{\left|\varphi_{\lambda}(\theta)\right|^{2}} d \theta_{2} \leq \frac{1}{2 \pi} \int_{-\infty}^{\infty} \frac{1}{\left|\varphi_{\lambda}(\theta)\right|^{2}} d \theta_{2} \leq \frac{64}{\left(E\left[X_{2}^{2}\right]\right)^{2}} K^{-3 / 4}$. 
Proof. By (3.9), we obtain that, for $\left|\theta_{1}\right| \leq r_{0} / 2$,

$$
\int_{-r_{0} / 2}^{r_{0} / 2} \frac{\theta_{2}^{2}}{\left|\varphi_{\lambda}(\theta)\right|^{2}} d \theta_{2} \leq \frac{4}{c_{*}} \int_{-r_{0} / 2}^{r_{0} / 2} \frac{\Re\left[\varphi_{\lambda}(\theta)\right]}{\left|\varphi_{\lambda}(\theta)\right|^{2}} d \theta_{2} \leq \frac{4}{c_{*}} \int_{-\infty}^{\infty} \Re\left[\frac{1}{\varphi_{\lambda}(\theta)}\right] d \theta_{2} .
$$

Assume that $2 D-A C \neq 0$. In view of (A.5), the definition of $\tilde{a}_{\lambda}\left(\theta_{1}\right)$ and $4 B-A^{2}+C^{2} \leq$ $\sqrt{K}$ imply that

$$
\int_{-\infty}^{\infty} \Re\left[\frac{1}{\varphi_{\lambda}(\theta)}\right] d \theta_{2}=2 \pi \tilde{a}_{\lambda}\left(\theta_{1}\right) \leq \frac{4 \pi}{\lambda E\left[X_{2}^{2}\right]} K^{-1 / 4}
$$

The above two inequalities give the desired estimate (i) in the case $2 D-A C \neq 0$. The proof of (i) in the case $2 D-A C=0$ is similar and is omitted.

Since $1 /\left|\varphi_{\lambda}(\theta)\right|^{2} \geq 0$, the first inequality of (ii) is clear. To obtain the last inequality of (ii), we calculate the integral of $1 /\left|\varphi_{\lambda}(\theta)\right|^{2}$ over $(-\infty, \infty)$ with respect to $\theta_{2}$. Assume $2 D-A C \neq 0$. The partial-fraction decomposition of $1 /\left|\varphi_{\lambda}(\theta)\right|^{2}$ is

$$
\frac{1}{\left|\varphi_{\lambda}(\theta)\right|^{2}}=\frac{4}{\left(\lambda E\left[X_{2}^{2}\right]\right)^{2}}\left(\frac{\bar{F} \theta_{2}+\bar{G}}{\theta_{2}^{2}+a_{+} \theta_{2}+b_{+}}+\frac{-\bar{F} \theta_{2}+\bar{I}}{\theta_{2}^{2}+a_{-} \theta_{2}+b_{-}}\right),
$$

where $M=\left(a_{+}-a_{-}\right)\left(a_{+} b_{-}-a_{-} b_{+}\right)+\left(b_{-}-b_{+}\right)^{2}$ as in (A.1) and

$$
\begin{aligned}
& \bar{F}=\frac{a_{+}-a_{-}}{M}, \\
& \bar{G}=\frac{a_{+}\left(a_{+}-a_{-}\right)+\left(b_{-}-b_{+}\right)}{M}, \\
& \bar{I}=\frac{-a_{-}\left(a_{+}-a_{-}\right)-\left(b_{-}-b_{+}\right)}{M} .
\end{aligned}
$$

This gives the integral

$$
\frac{\left(\lambda E\left[X_{2}^{2}\right]\right)^{2}}{4} \int_{-\infty}^{\infty} \frac{1}{\left|\varphi_{\lambda}(\theta)\right|^{2}} d \theta_{2}=\left(\frac{-a_{+} \bar{F}+2 \bar{G}}{\sqrt{4 b_{+}-a_{+}^{2}}}+\frac{a_{-} \bar{F}+2 \bar{I}}{\sqrt{4 b_{-}-a_{-}^{2}}}\right) \pi .
$$

A simple calculation and (A.1) show that the right-hand side of the above equality is equal to

$$
c_{\lambda}\left(\theta_{1}\right):=\left\{\frac{2 H-J_{2}}{\sqrt{J_{1}+J_{2}}}+\frac{2 H+J_{2}}{\sqrt{J_{1}-J_{2}}}\right\} \times \frac{2 \pi}{\left(-4 B+A^{2}+C^{2}+\sqrt{K}\right) \sqrt{K}} .
$$

We compute $\left(c_{\lambda}\left(\theta_{1}\right)\right)^{2}$ by using (A.3). From this computation and $c_{\lambda}\left(\theta_{1}\right) \geq 0$, we have

$$
c_{\lambda}\left(\theta_{1}\right)=\frac{4 \sqrt{2} \pi}{\sqrt{K}} \frac{\sqrt{4 B-A^{2}+C^{2}+\sqrt{K}}}{4 B-A^{2}-C^{2}+\sqrt{K}} .
$$

This implies the desired estimate (ii) in the case $2 D-A C \neq 0$. The proof of (ii) in the case $2 D-A C=0$ is similar and is omitted.

LemmA A.2. Assume that $(a)-(d)$ hold. Then there exists a constant $c>0$ such that, for $\lambda \in(1 / 2,1)$ and $\theta_{1} \in[-\pi, \pi]$ with $\left|\theta_{1}\right| \leq r_{0} / 2$,

(iii) $\frac{1}{2 \pi} \int_{-r_{0} / 2}^{r_{0} / 2} \frac{\left|\theta_{2}\right|^{2+\delta}}{\left|\varphi_{\lambda}(\theta)\right|^{2}} d \theta_{2} \leq c K^{(-1+\delta) / 4}(1+|\log K|)$, 
(iv) $\frac{1}{2 \pi} \int_{-r_{0} / 2}^{r_{0} / 2} \frac{\left|\theta_{2}\right|^{\gamma}}{\left|\varphi_{\lambda}(\theta)\right|^{2}} d \theta_{2} \leq c K^{(-3+\gamma) / 4} \quad(\gamma=\delta, 1,1+\delta)$.

Proof. We prove (iii). Since $\delta \in(0,1)$,

$$
\left|\theta_{2}\right|^{2+\delta} \leq \begin{cases}K^{(2+\delta) / 4}, & \left|\theta_{2}\right| \leq K^{1 / 4} \\ \left|\theta_{2}\right|^{3} K^{(-1+\delta) / 4}, & \left|\theta_{2}\right|>K^{1 / 4}\end{cases}
$$

We use (A.10) to obtain that the left-hand side of (iii) is bounded by

$$
\frac{K^{(2+\delta) / 4}}{2 \pi} \int_{-r_{0} / 2}^{r_{0} / 2} \frac{1}{\left|\varphi_{\lambda}(\theta)\right|^{2}} d \theta_{2}+\frac{K^{(-1+\delta) / 4}}{2 \pi} \int_{K^{1 / 4}<\left|\theta_{2}\right| \leq r_{0} / 2} \frac{\left|\theta_{2}\right|^{3}}{\left|\varphi_{\lambda}(\theta)\right|^{2}} d \theta_{2} .
$$

By Lemma A.1(ii), the first term of (A.11) is bounded by a constant multiple of $K^{(-1+\delta) / 4}$. From (3.9),

$$
\frac{\left|\theta_{2}\right|^{3}}{\left|\varphi_{\lambda}(\theta)\right|^{2}} \leq \frac{16}{c_{*}^{2}\left|\theta_{2}\right|} \quad\left(|\theta|<r_{0}, \lambda \in(1 / 2,1)\right)
$$

and the integral of $1 /\left|\theta_{2}\right|$ over $K^{1 / 4}<\left|\theta_{2}\right| \leq r_{0} / 2$ with respect to $\theta_{2}$ is bounded by $\left|\log \left(r_{0} / 2\right)\right|+(1 / 4)|\log K|$. The second term of (A.11) is bounded by a constant multiple of $K^{(-1+\delta) / 4}(1+|\log K|)$. Thus, we conclude that (iii) holds.

To prove (iv), we use the inequality

$$
\left|\theta_{2}\right|^{\gamma} \leq \begin{cases}K^{\gamma / 4} & \left(\left|\theta_{2}\right| \leq K^{1 / 4}\right) \\ \left|\theta_{2}\right|^{2} K^{(\gamma-2) / 4} & \left(\left|\theta_{2}\right|>K^{1 / 4}\right)\end{cases}
$$

instead of (A.10), and the result follows from Lemma A.1(i) and (ii).

Proof of Lemma 3.3. With $r_{0}>0$,

$$
\begin{aligned}
a_{\lambda}\left(\theta_{1}\right)-\frac{1}{2 \pi} \int_{-r_{0} / 2}^{r_{0} / 2} \mathfrak{R}\left[\frac{1}{\varphi_{\lambda}(\theta)}\right] d \theta_{2}= & \frac{1}{2 \pi} \int_{-r_{0} / 2}^{r_{0} / 2} \chi_{\lambda}(\theta) d \theta_{2} \\
& +\frac{1}{2 \pi} \int_{r_{0} / 2<\left|\theta_{2}\right| \leq \pi} \frac{\Re[1-\lambda \phi(\theta)]}{|1-\lambda \phi(\theta)|^{2}} d \theta_{2},
\end{aligned}
$$

where

$$
\chi_{\lambda}(\theta)=\frac{\mathfrak{R}[1-\lambda \phi(\theta)]}{|1-\lambda \phi(\theta)|^{2}}-\frac{\mathfrak{R}\left[\varphi_{\lambda}(\theta)\right]}{\left|\varphi_{\lambda}(\theta)\right|^{2}} .
$$

Equation (3.7) implies that, for $\lambda \in(1 / 2,1)$ and $\theta \in[-\pi, \pi]^{2}$ with $\left|\theta_{2}\right| \geq r_{0} / 2$,

$$
0 \leq \frac{\mathfrak{R}[1-\lambda \phi(\theta)]}{|1-\lambda \phi(\theta)|^{2}} \leq \frac{1}{|1-\lambda \phi(\theta)|} \leq \frac{2}{c_{*}|\theta|^{2}} \leq \frac{8}{c_{*} r_{0}^{2}} .
$$

Hence, the absolute value of the second term on the right-hand side of (A.12) is bounded by a constant.

To estimate the first term on the right-hand side of (A.12), we consider $\chi_{\lambda}(\theta)$. By applying the estimates (3.6), (3.8) and

$$
\frac{1}{|1-\lambda \phi(\theta)|} \leq \frac{c_{3}\left(\pi^{\hat{\delta}}+\pi^{\delta}\right)+1}{\left|\varphi_{\lambda}(\theta)\right|} \quad\left(\lambda \in(1 / 2,1), \theta \in[-\pi, \pi]^{2}\right),
$$


which follows from (3.1), we deduce that

$$
\begin{aligned}
\left|\chi_{\lambda}(\theta)\right| \leq & \left|\Re[1-\lambda \phi(\theta)]-\Re\left[\varphi_{\lambda}(\theta)\right]\right| \frac{1}{|1-\lambda \phi(\theta)|^{2}} \\
& +\left|\Re\left[\varphi_{\lambda}(\theta)\right]\right| \frac{\left|1-\lambda \phi(\theta)-\varphi_{\lambda}(\theta)\right|}{|1-\lambda \phi(\theta)|\left|\varphi_{\lambda}(\theta)\right|}\left(\frac{1}{|1-\lambda \phi(\theta)|}+\frac{1}{\left|\varphi_{\lambda}(\theta)\right|}\right) \\
\leq & c_{19} \frac{\left(\left|\theta_{1}\right|^{\hat{\delta}}+\left|\theta_{2}\right|^{\delta}\right) \theta_{2}^{2}}{\left|\varphi_{\lambda}(\theta)\right|^{2}},
\end{aligned}
$$

where $c_{19}$ is a constant independent of $\lambda \in(1 / 2,1)$ and $\theta \in[-\pi, \pi]^{2}$.

The following estimates complete the proof. The integral of $\left|\theta_{2}\right|^{2} /\left|\varphi_{\lambda}(\theta)\right|^{2}$ appearing on the right-hand side of the above inequality over $\left[-r_{0} / 2, r_{0} / 2\right]$ with respect to $\theta_{2}$ is bounded by a constant multiple of $K^{-1 / 4}$. Moreover, the integral of $\left|\theta_{2}\right|^{2+\delta} /\left|\varphi_{\lambda}(\theta)\right|^{2}$ appearing on the right-hand side of the above inequality over $\left[-r_{0} / 2, r_{0} / 2\right]$ with respect to $\theta_{2}$ is bounded by a constant multiple of $K^{(-1+\delta) / 4}|\log K|$. These estimates come from Lemma A.1(i) and Lemma A.2(iii). Note that $K$ tends to zero as $\theta_{1} \rightarrow 0$ and $r \uparrow 1$, which implies that $1 \leq|\log K|$.

Proof of Lemma 3.4. Equation (3.11) can be proved by the same procedure as in Lemma 3.2. We only give a sketch of the proof of (3.11) in the case $2 D-A C \neq 0$.

Assume that $2 D-A C \neq 0$. We calculate

$$
\Im\left[\frac{1}{2 \pi} \int_{-r_{0} / 2}^{r_{0} / 2} \frac{1}{\varphi_{\lambda}(\theta)} d \theta_{2}\right]=\frac{1}{2 \pi} \int_{-r_{0} / 2}^{r_{0} / 2} \Im\left[\frac{1}{\varphi_{\lambda}(\theta)}\right] d \theta_{2} .
$$

The partial-fraction decomposition of $\Im\left[1 / \varphi_{\lambda}(\theta)\right]$ is

$$
\Im\left[\frac{1}{\varphi_{\lambda}(\theta)}\right]=\frac{-\Im\left[\varphi_{\lambda}(\theta)\right]}{\left|\varphi_{\lambda}(\theta)\right|^{2}}=\frac{1}{(\lambda / 2) E\left[X_{2}^{2}\right]}\left(\frac{\tilde{F} \theta_{2}+\tilde{G}}{\theta_{2}^{2}+a_{+} \theta_{2}+b_{+}}+\frac{-\tilde{F} \theta_{2}+\tilde{I}}{\theta_{2}^{2}+a_{-} \theta_{2}+b_{-}}\right),
$$

where $M=\left(a_{+}-a_{-}\right)\left(a_{+} b_{-}-a_{-} b_{+}\right)+\left(b_{-}-b_{+}\right)^{2}$ as in (A.1) and

$$
\begin{aligned}
& \tilde{F}=\frac{C\left(b_{-}-b_{+}\right)+D\left(a_{+}-a_{-}\right)}{M}, \\
& \tilde{G}=\frac{-C\left(a_{+}-a_{-}\right) b_{+}+D a_{+}\left(a_{+}-a_{-}\right)+D\left(b_{-}-b_{+}\right)}{M}, \\
& \tilde{I}=\frac{C\left(a_{+}-a_{-}\right) b_{-}-D a_{-}\left(a_{+}-a_{-}\right)-D\left(b_{-}-b_{+}\right)}{M} .
\end{aligned}
$$

This gives the integral

$$
\begin{aligned}
& \frac{\lambda E\left[X_{2}^{2}\right]}{2} \int_{-r_{0} / 2}^{r_{0} / 2} \Im\left[\frac{1}{\varphi_{\lambda}(\theta)}\right] d \theta_{2} \\
& =\frac{\tilde{F}}{2}\left[\log \frac{\theta_{2}^{2}+a_{+} \theta_{2}+b_{+}}{\theta_{2}^{2}+a_{-} \theta_{2}+b_{-}}\right]_{-r_{0} / 2}^{r_{0} / 2}+\frac{-a_{+} \tilde{F}+2 \tilde{G}}{\sqrt{4 b_{+}-a_{+}^{2}}}\left[\arctan \frac{2 \theta_{2}+a_{+}}{\sqrt{4 b_{+}-a_{+}^{2}}}\right]_{-r_{0} / 2}^{r_{0} / 2} \\
& \quad+\frac{a_{-} \tilde{F}+2 \tilde{I}}{\sqrt{4 b_{-}-a_{-}^{2}}}\left[\arctan \frac{2 \theta_{2}+a_{-}}{\sqrt{4 b_{-}-a_{-}^{2}}}\right]_{-r_{0} / 2}^{r_{0} / 2}
\end{aligned}
$$


Using

$$
\tilde{F}=\frac{2 D-A C}{\sqrt{K} \sqrt{H}}, \quad|\log (1+x)| \leq 2|x| \quad(|x| \leq 1 / 2),
$$

and

$$
(2 D-A C)^{2}=\frac{1}{2}\left(4 B-A^{2}+C^{2}+\sqrt{K}\right) H,
$$

the first term on the right-hand side of (A.14) is bounded by a constant multiple of $|2 D-A C| / \sqrt{K}+|C|$. A simple calculation gives

$$
-a_{+} \tilde{F}+2 \tilde{G}=\frac{2 D-A C}{\sqrt{K}}-\frac{C \sqrt{H}}{\sqrt{K}}, \quad a_{-} \tilde{F}+2 \tilde{I}=\frac{2 D-A C}{\sqrt{K}}+\frac{C \sqrt{H}}{\sqrt{K}} .
$$

The difference between the second term on the right-hand side of (A.14) and

$$
\frac{-a_{+} \tilde{F}+2 \tilde{G}}{\sqrt{4 b_{+}-a_{+}^{2}}} \pi=\frac{\pi}{\sqrt{J_{1}+J_{2}} \sqrt{K}}(2 D-A C-C \sqrt{H})
$$

is bounded by a constant multiple of $|2 D-A C| / \sqrt{K}+|C| / K^{1 / 4}$. Similarly, the difference between the third term on the right-hand side of (A.14) and

$$
\frac{a_{-} \tilde{F}+2 \tilde{I}}{\sqrt{4 b_{-}-a_{-}^{2}}} \pi=\frac{\pi}{\sqrt{J_{1}-J_{2}} \sqrt{K}}(2 D-A C+C \sqrt{H})
$$

is bounded by a constant multiple of $|2 D-A C| / \sqrt{K}+|C| / K^{1 / 4}$.

Put

$$
\bar{b}_{\lambda}\left(\theta_{1}\right)=\frac{1}{\lambda E\left[X_{2}^{2}\right] \sqrt{K}}\left(\frac{2 D-A C-C \sqrt{H}}{\sqrt{J_{1}+J_{2}}}+\frac{2 D-A C+C \sqrt{H}}{\sqrt{J_{1}-J_{2}}}\right) .
$$

Then the difference between $(1 / 2 \pi) \int_{-r_{0} / 2}^{r_{0} / 2} \Im\left[1 / \varphi_{\lambda}(\theta)\right] d \theta_{2}$ and $\bar{b}_{\lambda}\left(\theta_{1}\right)$ is bounded by a constant multiple of $|2 D-A C| / \sqrt{K}+|C| / K^{1 / 4}$.

It remains to show that

$$
\bar{b}_{\lambda}\left(\theta_{1}\right)=\tilde{b}_{\lambda}\left(\theta_{1}\right):=\frac{\sqrt{2}}{\lambda E\left[X_{2}^{2}\right] \sqrt{K}} \frac{2(2 D-A C)}{\sqrt{4 B-A^{2}+C^{2}+\sqrt{K}}} .
$$

By using (A.3) and noticing (A.15), we have

$$
\bar{b}_{\lambda}^{2}\left(\theta_{1}\right)=\frac{4 H}{\left(\lambda E\left[X_{2}^{2}\right]\right)^{2} K}=\tilde{b}_{\lambda}^{2}\left(\theta_{1}\right) .
$$

From the inequality

$$
0 \leq C^{2} H \leq \frac{1}{2}\left(4 B-A^{2}+C^{2}+\sqrt{K}\right) H=(2 D-A C)^{2},
$$

the sign of $\tilde{b}_{\lambda}\left(\theta_{1}\right)$ is the same as that of $\bar{b}_{\lambda}\left(\theta_{1}\right)$. Equation (A.16) yields $\bar{b}_{\lambda}\left(\theta_{1}\right)=\tilde{b}_{\lambda}\left(\theta_{1}\right)$. 
Proof of Lemma 3.5. Let $\hat{b}_{\lambda}\left(\theta_{1}\right)$ be the imaginary part of

$$
\frac{1}{2 \pi} \int_{-r_{0} / 2}^{r_{0} / 2} \frac{1}{\varphi_{\lambda}\left(\theta_{1}, \theta_{2}\right)} d \theta_{2}
$$

By performing the change of variables $\theta_{2}=-\tilde{\theta}_{2}$ and using the symmetry of trigonometric functions, $b_{\lambda}\left(\theta_{1}\right)=-b_{\lambda}\left(-\theta_{1}\right)$ and $\hat{b}_{\lambda}\left(\theta_{1}\right)=-\hat{b}_{\lambda}\left(-\theta_{1}\right)$. In view of these equalities,

$$
\begin{aligned}
b_{\lambda}\left(\theta_{1}\right)-\hat{b}_{\lambda}\left(\theta_{1}\right)= & \frac{1}{2}\left\{b_{\lambda}\left(\theta_{1}\right)-b_{\lambda}\left(-\theta_{1}\right)-\left(\hat{b}_{\lambda}\left(\theta_{1}\right)-\hat{b}_{\lambda}\left(-\theta_{1}\right)\right)\right\} \\
= & \frac{1}{4 \pi} \int_{-r_{0} / 2}^{r_{0} / 2} \chi_{\lambda}^{(1)}\left(\theta_{1}, \theta_{2}\right) d \theta_{2}+\frac{1}{4 \pi} \int_{-r_{0} / 2}^{r_{0} / 2} \chi_{\lambda}^{(2)}\left(\theta_{1}, \theta_{2}\right) d \theta_{2} \\
& \quad+\frac{1}{4 \pi} \int_{r_{0} / 2 \leq\left|\theta_{2}\right| \leq \pi} \chi_{\lambda}^{(3)}\left(\theta_{1}, \theta_{2}\right) d \theta_{2},
\end{aligned}
$$

where

$$
\begin{aligned}
\chi_{\lambda}^{(1)}\left(\theta_{1}, \theta_{2}\right)= & \lambda E\left[\left(\sin \theta_{1} X_{1}\right)\left(\cos \theta_{2} X_{2}\right)\right]\left(\frac{1}{\left|1-\lambda \phi\left(\theta_{1}, \theta_{2}\right)\right|^{2}}+\frac{1}{\left|1-\lambda \phi\left(-\theta_{1}, \theta_{2}\right)\right|^{2}}\right) \\
& -\lambda E\left[\sin \theta_{1} X_{1}\right]\left(\frac{1}{\left|\varphi_{\lambda}\left(\theta_{1}, \theta_{2}\right)\right|^{2}}+\frac{1}{\left|\varphi_{\lambda}\left(-\theta_{1}, \theta_{2}\right)\right|^{2}}\right), \\
\chi_{\lambda}^{(2)}\left(\theta_{1}, \theta_{2}\right)= & \lambda E\left[\left(\cos \theta_{1} X_{1}\right)\left(\sin \theta_{2} X_{2}\right)\right]\left(\frac{1}{\left|1-\lambda \phi\left(\theta_{1}, \theta_{2}\right)\right|^{2}}-\frac{1}{\left|1-\lambda \phi\left(-\theta_{1}, \theta_{2}\right)\right|^{2}}\right) \\
& -\lambda E\left[\left(-1+\cos \theta_{1} X_{1}\right) X_{2}\right] \theta_{2}\left(\frac{1}{\left|\varphi_{\lambda}\left(\theta_{1}, \theta_{2}\right)\right|^{2}}-\frac{1}{\left|\varphi_{\lambda}\left(-\theta_{1}, \theta_{2}\right)\right|^{2}}\right)
\end{aligned}
$$

and

$$
\chi_{\lambda}^{(3)}\left(\theta_{1}, \theta_{2}\right)=\frac{\lambda E\left[\sin \left(\theta_{1} X_{1}+\theta_{2} X_{2}\right)\right]}{\left|1-\lambda \phi\left(\theta_{1}, \theta_{2}\right)\right|^{2}}-\frac{\lambda E\left[\sin \left(-\theta_{1} X_{1}+\theta_{2} X_{2}\right)\right]}{\left|1-\lambda \phi\left(-\theta_{1}, \theta_{2}\right)\right|^{2}} .
$$

Using condition (c),

$$
\begin{aligned}
\left|\phi\left(\theta_{1}, \theta_{2}\right)-\phi\left(-\theta_{1}, \theta_{2}\right)\right| & \leq 2\left|E\left[\left(\sin \theta_{1} X_{1}\right)\left(\sin \theta_{2} X_{2}\right)\right]\right|+2 \mid E\left[\left(\sin \theta_{1} X_{1}\right)\left(\cos \theta_{2} X_{2}\right)\right] \\
& \leq 4 E\left[\left|\sin \theta_{1} X_{1}\right|\right] \leq 4 E\left[\left|X_{1}\right|^{\delta}\right]\left|\theta_{1}\right|^{\delta} .
\end{aligned}
$$

The above inequalities and (3.7) imply that, for $\lambda \in(1 / 2,1)$ and $\left(\theta_{1}, \theta_{2}\right) \in[-\pi, \pi] \times$ $[-\pi, \pi]$ with $\left|\theta_{2}\right| \geq r_{0} / 2$,

$$
\begin{aligned}
\left|\chi_{\lambda}^{(3)}\left(\theta_{1}, \theta_{2}\right)\right| \leq & \frac{\left|E\left[\left(\sin \theta_{1} X_{1}\right)\left(\cos \theta_{2} X_{2}\right)\right]\right|}{\left|1-\lambda \phi\left(\theta_{1}, \theta_{2}\right)\right|^{2}}+\frac{\left|E\left[\left(\sin \theta_{1} X_{1}\right)\left(\cos \theta_{2} X_{2}\right)\right]\right|}{\left|1-\lambda \phi\left(-\theta_{1}, \theta_{2}\right)\right|^{2}} \\
& +\left|E\left[\left(\cos \theta_{1} X_{1}\right)\left(\sin \theta_{2} X_{2}\right)\right]\right|\left|\frac{1}{\left|1-\lambda \phi\left(\theta_{1}, \theta_{2}\right)\right|^{2}}-\frac{1}{\left|1-\lambda \phi\left(-\theta_{1}, \theta_{2}\right)\right|^{2}}\right| \\
\leq & \frac{E\left[\left|X_{1}\right|^{\delta}\right]\left|\theta_{1}\right|^{\delta}}{\left|1-\lambda \phi\left(\theta_{1}, \theta_{2}\right)\right|^{2}}+\frac{E\left[\left|X_{1}\right|^{\delta}\right]\left|\theta_{1}\right|^{\delta}}{\left|1-\lambda \phi\left(-\theta_{1}, \theta_{2}\right)\right|^{2}} \\
& +\left|\phi\left(\theta_{1}, \theta_{2}\right)-\phi\left(-\theta_{1}, \theta_{2}\right)\right| \frac{\left|1-\lambda \phi\left(-\theta_{1}, \theta_{2}\right)\right|+\left|1-\lambda \phi\left(\theta_{1}, \theta_{2}\right)\right|}{\left|1-\lambda \phi\left(\theta_{1}, \theta_{2}\right)\right|^{2}\left|1-\lambda \phi\left(-\theta_{1}, \theta_{2}\right)\right|^{2}} \\
\leq & \frac{128 E\left[\left|X_{1}\right|^{\delta}\right]}{c_{*}^{2} r_{0}^{4}}\left(1+\frac{32}{c_{*} r_{0}^{2}}\right)\left|\theta_{1}\right|^{\delta} .
\end{aligned}
$$


Hence, the third term on the right-hand side of the last equality in (A.17) is bounded by a constant multiple of $\left|\theta_{1}\right|^{\delta}$.

To estimate remaining terms on the right-hand side of the last equality in (A.17), we consider $\chi_{\lambda}^{(1)}\left(\theta_{1}, \theta_{2}\right)$ and $\chi_{\lambda}^{(2)}\left(\theta_{1}, \theta_{2}\right)$. With the help of

$$
\left|E\left[\left(\sin \theta_{1} X_{1}\right)\left(\cos \theta_{2} X_{2}\right)\right]-E\left[\sin \theta_{1} X_{1}\right]\right| \leq E\left[\left|X_{1}\right|^{\hat{\delta}} X_{2}^{2}\right]\left|\theta_{1}\right|^{\hat{\delta}} \theta_{2}^{2}
$$

and (3.7), the same argument as shown in (A.13) implies that

$$
\begin{aligned}
\left|\chi_{\lambda}^{(1)}\left(\theta_{1}, \theta_{2}\right)\right| \leq & \frac{\left|E\left[\left(\sin \theta_{1} X_{1}\right)\left(\cos \theta_{2} X_{2}\right)\right]-E\left[\sin \theta_{1} X_{1}\right]\right|}{\left|1-\lambda \phi\left(\theta_{1}, \theta_{2}\right)\right|^{2}} \\
& +\frac{\left|E\left[\left(\sin \theta_{1} X_{1}\right)\left(\cos \theta_{2} X_{2}\right)\right]-E\left[\sin \theta_{1} X_{1}\right]\right|}{\left|1-\lambda \phi\left(-\theta_{1}, \theta_{2}\right)\right|^{2}} \\
& +\left|E\left[\sin \theta_{1} X_{1}\right]\right| \frac{\left|1-\lambda \phi\left(\theta_{1}, \theta_{2}\right)-\varphi_{\lambda}\left(\theta_{1}, \theta_{2}\right)\right|}{\left|1-\lambda \phi\left(\theta_{1}, \theta_{2}\right)\right|\left|\varphi_{\lambda}\left(\theta_{1}, \theta_{2}\right)\right|} \\
& \times\left(\frac{1}{\left|1-\lambda \phi\left(\theta_{1}, \theta_{2}\right)\right|}+\frac{1}{\left|\varphi_{\lambda}\left(\theta_{1}, \theta_{2}\right)\right|}\right) \\
& +\left|E\left[\sin \theta_{1} X_{1}\right]\right| \frac{\left|1-\lambda \phi\left(-\theta_{1}, \theta_{2}\right)-\varphi_{\lambda}\left(-\theta_{1}, \theta_{2}\right)\right|}{\left|1-\lambda \phi\left(-\theta_{1}, \theta_{2}\right)\right|\left|\varphi_{\lambda}\left(-\theta_{1}, \theta_{2}\right)\right|} \\
& \times\left(\frac{1}{\left|1-\lambda \phi\left(-\theta_{1}, \theta_{2}\right)\right|}+\frac{1}{\left|\varphi_{\lambda}\left(-\theta_{1}, \theta_{2}\right)\right|}\right) \\
\leq & c_{20}\left\{|D|\left(\left|\theta_{1}\right|^{\hat{\delta}}+\left|\theta_{2}\right|^{\delta}\right)+\left|\theta_{1}\right|^{\hat{\delta}} \theta_{2}^{2}\right\}\left(\frac{1}{\left|\varphi_{\lambda}\left(\theta_{1}, \theta_{2}\right)\right|^{2}}+\frac{1}{\left|\varphi_{\lambda}\left(-\theta_{1}, \theta_{2}\right)\right|^{2}}\right),
\end{aligned}
$$

where $c_{20}$ is a constant independent of $\lambda \in(1 / 2,1)$ and $\left(\theta_{1}, \theta_{2}\right) \in[-\pi, \pi] \times[-\pi, \pi]$.

By performing the change of variables $\theta_{2}=-\tilde{\theta}_{2}$ and using the symmetry of trigonometric functions,

$$
\int_{-r_{0} / 2}^{r_{0} / 2} \frac{\left|\theta_{2}\right|^{\gamma}}{\left|\varphi_{\lambda}\left(-\theta_{1}, \theta_{2}\right)\right|^{2}} d \theta_{2}=\int_{-r_{0} / 2}^{r_{0} / 2} \frac{\left|\tilde{\theta}_{2}\right|^{\gamma}}{\left|\varphi_{\lambda}\left(\theta_{1}, \tilde{\theta}_{2}\right)\right|^{2}} d \tilde{\theta}_{2} \quad(\gamma \geq 0) .
$$

Taking note of (A.19), it follows from (A.18), Lemma A.1 and Lemma A.2(iv) that the first term on the right-hand side of the last equality in (A.17) is bounded by a constant multiple of $\left|\theta_{1}\right|^{\hat{\delta}} K^{-1 / 4}+|D|\left(\left|\theta_{1}\right|^{\hat{\delta}}+K^{\delta / 4}\right) K^{-3 / 4}$.

From conditions (b), (c) and (d), we obtain

$$
\begin{aligned}
& \left|E\left[\left(\cos \theta_{1} X_{1}\right)\left(\sin \theta_{2} X_{2}\right)\right]-E\left[\left(-1+\cos \theta_{1} X_{1}\right) X_{2}\right] \theta_{2}\right| \\
& \quad=\left|E\left[\left(\cos \theta_{1} X_{1}\right)\left(\sin \theta_{2} X_{2}\right)\right]-E\left[\left(\cos \theta_{1} X_{1}\right) X_{2}\right] \theta_{2}\right| \leq E\left[\left|X_{2}\right|^{2+\delta}\right]\left|\theta_{2}\right|^{2+\delta}
\end{aligned}
$$

and

$\left|\phi\left(\theta_{1}, \theta_{2}\right)-\phi\left(-\theta_{1}, \theta_{2}\right)\right| \leq 2\left|E\left[\sin \theta_{1} X_{1}\right]\right|+2\left|E\left[\left(\sin \theta_{1} X_{1}\right) X_{2}\right]\right|\left|\theta_{2}\right|+2 E\left[\left|X_{1}\right|^{\hat{\delta}} X_{2}^{2}\right]\left|\theta_{1}\right|^{\hat{\delta}} \theta_{2}^{2}$. 
Using the above two inequalities and (3.7), the same argument as given in (A.13) implies that

$$
\begin{aligned}
\left|\chi_{\lambda}^{(2)}\left(\theta_{1}, \theta_{2}\right)\right| \leq & \frac{\left|E\left[\left(\cos \theta_{1} X_{1}\right)\left(\sin \theta_{2} X_{2}\right)\right]-E\left[\left(-1+\cos \theta_{1} X_{1}\right) X_{2}\right] \theta_{2}\right|}{\left|1-\lambda \phi\left(\theta_{1}, \theta_{2}\right)\right|\left|1-\lambda \phi\left(-\theta_{1}, \theta_{2}\right)\right|} \\
& \times\left|\phi\left(\theta_{1}, \theta_{2}\right)-\phi\left(-\theta_{1}, \theta_{2}\right)\right|\left(\frac{1}{\left|1-\lambda \phi\left(\theta_{1}, \theta_{2}\right)\right|}+\frac{1}{\left|1-\lambda \phi\left(-\theta_{1}, \theta_{2}\right)\right|}\right) \\
& +\left|E\left[\left(-1+\cos \theta_{1} X_{1}\right) X_{2}\right] \theta_{2}\right| \frac{\left|1-\lambda \phi\left(\theta_{1}, \theta_{2}\right)-\varphi_{\lambda}\left(\theta_{1}, \theta_{2}\right)\right|}{\left|1-\lambda \phi\left(\theta_{1}, \theta_{2}\right)\right|\left|\varphi_{\lambda}\left(\theta_{1}, \theta_{2}\right)\right|} \\
& \times\left(\frac{1}{\left|1-\lambda \phi\left(\theta_{1}, \theta_{2}\right)\right|}+\frac{1}{\left|\varphi_{\lambda}\left(\theta_{1}, \theta_{2}\right)\right|}\right) \\
& +\left|E\left[\left(-1+\cos \theta_{1} X_{1}\right) X_{2}\right] \theta_{2}\right| \frac{\left|1-\lambda \phi\left(-\theta_{1}, \theta_{2}\right)-\varphi_{\lambda}\left(-\theta_{1}, \theta_{2}\right)\right|}{\left|1-\lambda \phi\left(-\theta_{1}, \theta_{2}\right)\right|\left|\varphi_{\lambda}\left(-\theta_{1}, \theta_{2}\right)\right|} \\
& \times\left(\frac{1}{\left|1-\lambda \phi\left(-\theta_{1}, \theta_{2}\right)\right|}+\frac{1}{\left|\varphi_{\lambda}\left(-\theta_{1}, \theta_{2}\right)\right|}\right) \\
\leq & c_{21}\left\{|D|\left|\theta_{2}\right|^{\delta}+(|A|+|C|)\left|\theta_{2}\right|^{1+\delta}+\left(|C|\left|\theta_{2}\right|+\left|\theta_{2}\right|^{2}\right)\left|\theta_{1}\right|^{\delta}\right\} \\
& \times\left(\frac{1}{\left|\varphi_{\lambda}\left(\theta_{1}, \theta_{2}\right)\right|^{2}}+\frac{1}{\left|\varphi_{\lambda}\left(-\theta_{1}, \theta_{2}\right)\right|^{2}}\right),
\end{aligned}
$$

where $c_{21}$ is a constant independent of $\lambda \in(1 / 2,1)$ and $\left(\theta_{1}, \theta_{2}\right) \in[-\pi, \pi] \times[-\pi, \pi]$. Recalling (A.19), it follows from (A.20), Lemma A.1(i) and Lemma A.2(iv) that the second term on the right-hand side of the last equality in (A.17) is bounded by a constant multiple of $\left\{\left|\theta_{1}\right|^{\hat{\delta}}+|A| K^{(-1+\delta) / 4}+|C|\left(\left|\theta_{1}\right|^{\hat{\delta}}+K^{\delta / 4}\right) K^{-1 / 4}+|D| K^{(-2+\delta) / 4}\right\} K^{-1 / 4}$. Hence, we have the desired estimate.

\section{B. Appendix: Calculation of (1.4) and (1.5)}

To obtain (1.4) and (1.5), we use

$$
\int_{0}^{+\infty} \frac{\sin a x}{x^{\beta}} d x=\frac{\pi a^{\beta-1}}{2 \Gamma(\beta) \sin ((\beta \pi) / 2)}
$$

for $\beta \in(0,2)$ and $a>0$.

From the definition of $X_{1}$,

$$
1-E\left[\cos \vartheta X_{1}\right]=c_{+}(1-\cos \vartheta)+c_{-} \sum_{n=1}^{\infty}(1-\cos \vartheta) \frac{1}{n^{1+\alpha}} .
$$

Since the absolute value of the difference between the right-hand side of the above and

$$
c_{-} \int_{0}^{+\infty} \frac{1-\cos |\vartheta| x}{x^{1+\alpha}} d x
$$

is bounded by a constant multiple of $\vartheta^{2}$, it remains to show that

$$
c_{-} \int_{0}^{+\infty} \frac{1-\cos |\vartheta| x}{x^{1+\alpha}} d x=\frac{c_{-} \pi}{2 \Gamma(\alpha+1) \cos ((\alpha-1) \pi / 2)}|\vartheta|^{\alpha} .
$$


We perform integration by parts for the left-hand side of (B.2) and use (B.1). Then we have (B.2).

Noting $E\left[X_{1}\right]=0$,

$$
E\left[\sin \vartheta X_{1}\right]=c_{+}(\sin \vartheta-\vartheta)+c_{-} \sum_{n=1}^{\infty}(\vartheta n-\sin \vartheta n) \frac{1}{n^{1+\alpha}} .
$$

Since the absolute value of the difference between the right-hand side of the above and

$$
c_{-} \int_{0}^{+\infty} \frac{\vartheta x-\sin \vartheta x}{x^{1+\alpha}} d x
$$

is bounded by a constant multiple of $\vartheta^{2}$, it remains to show that

$$
c_{-} \int_{0}^{+\infty} \frac{\vartheta x-\sin \vartheta x}{x^{1+\alpha}} d x=\frac{c_{-} \pi}{2 \Gamma(\alpha+1) \sin ((\alpha-1) \pi / 2)}|\vartheta|^{\alpha} \frac{\vartheta}{|\vartheta|} .
$$

We perform integration by part twice for the left-hand side of (B.3) and use (B.1). Then we have (B.3).

\title{
REFERENCES
}

[1] M. Bousquet-Mélou and G. Schaeffer. Walks of the slit plane. Probab. Theory Related Fields 124 (2002), 305-344.

[2] Y. Fukai. Hitting time of a half-line by two-dimensional random walk. Probab. Theory Related Fields 128 (2004), 323-346.

[3] Y. Isozaki. Fluctuation identities applied to the hitting time of a half-line in the plane. J. Theoret. Probab. 22(1) (2009), 57-81.

[4] H. Kesten. Hitting probabilities of random walks on $Z^{d}$. Stoch. Process. Appl. 25 (1987), 165-184.

[5] G. F. Lawler. Intersections of Random Walks. Birkhäuser, Boston, MA, 1991.

[6] G. F. Lawler. A discrete analogue of a theorem of Makarov. Combin. Probab. Comput. 2 (1993), 181-199.

[7] F. Spitzer. Principles of Random Walk, 2nd edn. Springer, Berlin, 1976.

\author{
Yasunari Fukai \\ Faculty of Mathematics \\ Kyushu University \\ Nishi-Ku, Fukuoka 812-0395 \\ Japan \\ (E-mail:fukai@math.kyushu-u.ac.jp)
}

